\title{
NUMERICAL INVESTIGATION OF A WAVE GLIDER IN HEAD SEAS
}

Fuming Yang, School of Naval Architecture and Ocean Engineering, Harbin Institute of Technology, Weihai, PR China

Weichao Shi, Department of Naval Architecture, Ocean and Marine Engineering, University of Strathclyde, U.K.

Xu Zhou, School of Naval Architecture and Ocean Engineering, Harbin Institute of Technology, Weihai, PR China

Bin Guo, School of Naval Architecture and Ocean Engineering, Harbin Institute of Technology, Weihai, PR China

Dazheng Wang, School of Naval Architecture and Ocean Engineering, Harbin Institute of Technology, Weihai, PR China

*Corresponding author: Dazheng Wang

Telephone number: $\quad+\quad+8618363154418$

Address: School of Naval Architecture and Ocean Engineering, Harbin institute of technology, No.2, Western Wenhua Road, Shandong Province, China, 264209

E-mail address: dazheng.wang@hitwh.edu.cn 
Abstract: A wave glider comprises a surface boat, which harvests energy from wave and solar power, a submerged glider containing six pairs of tandem hydrofoils and a tether connecting them in between. This paper presents a numerical simulation to predict the wave glider dynamic performance in head seas with the aid of computational fluid dynamic (CFD) method. The simulation involves two commercial CFD software packages, FINE/Marine and STAR-CCM+.

Firstly, unsteady Reynolds Averaged Navier-Stokes (URANS) simulation was built in FINE/Marine with volume of fluid (VOF) model to simulate the flow around the surface boat and the tandem hydrofoils as a system, followed by the high-fidelity simulation of the passive eccentric rotation of the underwater tandem hydrofoils in STAR-CCM+ using overset mesh. By taking the advantages of both softwares, manual iteration was conducted to achieve a converged result. Consequently, by analyzing these results, the surge force acting on the surface boat and the passive eccentric rotation law of the hydrofoils have been achieved which are proved to be the main factors affecting the propulsion efficiency of the wave glider.

Keywords: wave glider, passive eccentric rotation, surge force 


\section{Nomenclature}

$\xi \quad$ is the displacement of the wave glider in the $x$ direction.

$\gamma \quad$ is the displacement of the wave glider in the $y$ direction.

$\zeta \quad$ is the displacement of the wave glider in the $y$ direction.

$\psi \quad$ is the yaw angle of the wave glider.

$\theta \quad$ is the pitch angle of the wave glider.

$\phi \quad$ is the roll angle of the wave glider.

$\xi_{b} \quad$ is the displacement of the surface boat in the $\mathrm{x}$ direction.

$\gamma_{b} \quad$ is the displacement of the surface boat in the $y$ direction.

$\zeta_{b} \quad$ is the displacement of the surface boat in the y direction.

$\psi_{b} \quad$ is the yaw angle of the surface boat.

$\theta_{b} \quad$ is the pitch angle of the surface boat.

$\phi_{b} \quad$ is the roll angle of the surface boat.

$\xi_{g} \quad$ is the displacement of the glider in the $\mathrm{x}$ direction.

$\gamma_{g} \quad$ is the displacement of the glider in the y direction.

$\zeta_{g} \quad$ is the displacement of the glider in the $y$ direction.

$\psi_{g} \quad$ is the yaw angle of the glider.

$\theta_{g} \quad$ is the pitch angle of the glider.

$\phi_{g} \quad$ is the roll angle of the glider.

$H \quad$ is the wave height.

$T \quad$ is the wave period.

$S_{f} \quad$ is the waterplane area of the surface boat.

$P_{W G i} \quad$ is the wave energy absorption power of the wave glider.

$P_{W G m} \quad$ is the mechanical conversion power of the wave glider.

$X_{W G}(t) \quad$ is the time-varying forces of the surface boat in the $\mathrm{x}$ direction.

$Z_{W G}(t) \quad$ is the time-varying forces of the surface boat in the $z$ direction.

$F_{W G} \quad$ is the time-averaged value of $X_{W G}(t)$. 


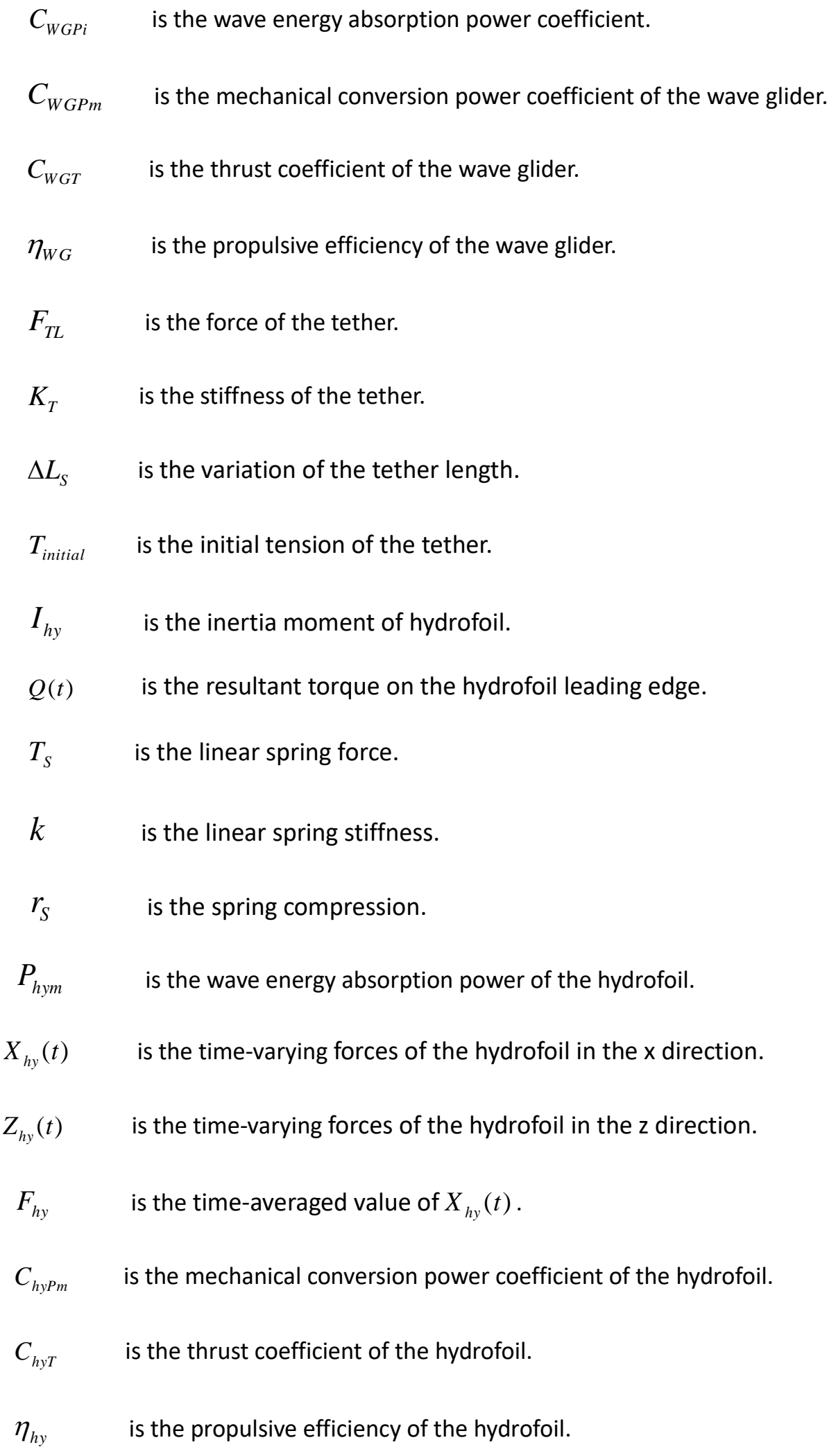




\section{Introduction}

Design and development of devices for oceanic research and atmospheric monitoring have drawn great attentions nowadays, especially for those meeting the requirement of lower-cost replacement and real-time communication worldwide. The first wave glider, developed by Liquid Robotics Corporation (Smith et al. (2011)), relies on the wave motion to propel the system to conduct surface as well as underwater missions. Therefore, it is crucial to understand the system's motion dynamics in the waves so that to achieve a better performance in the real seas.

The methodologies to predict the motion dynamics of the wave glider in the real seas can be either based on model-scale lab tests or numerical simulations. Model-scale lab tests are deemed to be the most effective way as well as the most expensive way due to the demand of highly specialized hydrodynamic testing facilities. With the benefit of conducting systematic investigations at a minimum cost, the later has often been the preferable option (Elhadad et al. (2014), Tian et al. (2014), Jia et al. (2014), Tian et al. (2015), Liu et al. (2016)). With the enhancement of the modern computational technology, numerical simulation using the unsteady Reynolds Averaged Navier-Stokes (URANS) is acknowledged to be an ideal solution to investigate the performance of the wave glider (Jia et al. (2014), Liu et al. (2016). Before this current study, the steady state CFD simulation based on RANS has been successfully applied to assess the velocity based thrust and drag coefficients of the wave glider. Jia et al. (2014) have made a comparison of the hydrodynamic results of NACA series' airfoil with plate wing under different flow velocities and various spacing by using CFD software ANSYS-Fluent. Elhadad et al. (2014) employed the Wigley model as the surface boat of Wave Glider and calculated the resistance characteristics at a range of Froude numbers, 0.10.4. Zheng et al. (2015) compared different factors influencing the drag force converted by the usage of NACA63-412 asymmetric airfoil and optimized them by making a comparison of the simulation results with the calculation results. However, the previous numerical simulation is often based on the steady simulation without considering the unsteady phenomenon nor the passive rotation of the wings; the multi-body dynamics of the wings was estimated via semi-empirical or empirical formulas. Ngo et al. (2013) applied the linear regression and Gauss regression model to discuss the environmental parameters that influence the movement of the wave glider and to predict the forward speed of the wave glider by inputting the wave parameters such as wave height, wave period, wind speed and current. Kraus (2012) established the sixdegree-of-freedom nonlinear dynamic equation to determine the key hydrodynamic parameters according 
to the Newton's law of momentum theorem and momentum moment theorem with the consideration of the influence of wind, flow and added mass. Baoqiang et al. (2014) concentrated on the movement efficiency of the wave glider. The model of movement efficiency was established from the perspective of energy conversion, and then this formula was further confirmed through direct comparison of the numerical results based on linear wave theory with experimental results. Furthermore, Tian et al. (2015) applied the D-H approach and the Lagrange mechanics to the simulation of the dynamic motion of the wave glider. From these simulations mentioned above, only the thrust and drag coefficients of the wave glider can be determined. In addition, there is lack of numerical simulation of the passive hydrodynamic rotation of the hydrofoils, which is essential to simulate the coupled motion of the surface boat and the glider.

The aim of the present study is to explore the feasibility of developing a high fidelity numerical simulation method to fully evaluate the motion characteristics of the wave glider in the waves. The simulations of only surface boat, the wave glider in head seas and the passive eccentric rotation of the hydrofoils are conducted using the URANS solver, all of which act to investigate the parameters that affect the motion of the wave glider. 


\section{Description of the wave glider}

The wave glider model used in this paper was developed by China National Marine Technology Center, originally manufactured by Liquid Robotics Corporation. As shown in Fig.1, there are three major components consisted in the wave glider: a surface boat housing sensors, a submerged glider containing six hinged flat hydrofoils which are arranged in tandem on both sides and a tether that connects the surface boat and the glider. The wave glider is symmetrical to its transversal midsection and the surface boat is also symmetrical to the longitudinal section in the center plane. A spring is introduced and mounted beneath each hydrofoil and the diagram is shown in Fig.2. The distance between adjacent row of tandem hydrofoils is $90 \mathrm{~mm}$. The chord of the hydrofoil is $160 \mathrm{~mm}$ and the rotational axis of the hydrofoil is placed $50 \mathrm{~mm}$ after the leading edge. The spring is wrapped in a sleeve and the neutral length is $120 \mathrm{~mm}$. One end of the spring sleeve is installed at a distance of $40 \mathrm{~mm}$ from the rotation axis; the other end is installed $17 \mathrm{~mm}$ below the baseline of the hydrofoil to form an angle between the baseline of the hydrofoil and the spring, 7.9 degrees. Therefore, when the hydrofoil rotates, the spring will slow down and limit the rotation of the hydrofoil to provide suitable angle of attack to produce the thrust. The oscillation angle of each hydrofoil is limited to $45^{\circ}$ to $60^{\circ}$. The general parameters for a full-scale sized wave glider is shown in Table 1.

As shown in Fig.3, when the surface boat rides over a wave, it pulls the glider up through the tether, which induces an angle of attack for the hydrofoils to generate a thrust to propel the system forward. Similarly, as the glider descends, the hydrofoils flap and produce another thrust force to propel the system. When the wave glider speed through water is lower than the surface boat, the hydrofoil remains horizontally neutral, no longer providing any thrust.

The six degree of freedom motion of the wave glider is normally described using three coordinate systems. The first coordinate system is an inertial reference frame fixed with respect to mean position of the surface boat which is defined for the hydrodynamic problem, $X=(x, y, z)$, with $z$ in the vertical direction, $x$ along the longitudinal direction of the surface boat and pointing to the bow, and $y$ perpendicular to the $x$ and pointing to in the port direction. The origin is in the plane of the undisturbed free surface. The other coordinate systems, body-fixed coordinate system, are the surface boat and the glider coordinate systems. The bodyfixed coordinate systems are parallel to the inertial coordinate system. The origins are in the longitudinal profile on their center of gravity, $1.363 \mathrm{~m}$ from the origin of inertial reference frame and $0.017 \mathrm{~m}$ above the 
waterplane for the surface boat while $1.361 \mathrm{~m}$ from the origin of the inertial reference frame and $3.444 \mathrm{~m}$ below the waterplane for the glider. In the body-fixed coordinate system, the position of the wave glider's coordinate system is then express in $\xi, \gamma, \zeta$ coordinates. The rotation of the body-fixed coordinate system is described by Euler angles $\psi$ (yaw), $\theta$ (pitch) and $\phi$ (roll) in Fig.4.

Considering the surface boat advancing in waves and oscillating as an unrestrained rigid body, the oscillatory motions consist of three translations and three rotations. Although theory is valid for arbitrary headings, the present work is restricted to only head seas. Hence the oscillatory motions to be studied are surge and heave motions. It is assumed that the pitch amplitude is small enough to be neglected. The rigid body motions are solved with respect to the inertial coordinate system that moves with the surface boat and the glider, respectively.

In order to make a comparison between the numerical simulation and the tank test, the hydrodynamic tank tests were performed in China National Marine Technology Center in their towing tank facility, length $\times$ width $\times$ depth $=130 \mathrm{~m} \times 7 \mathrm{~m} \times 5 \mathrm{~m}$. As shown in Fig.5, the free sailing test was conducted to test the sailing velocity of the wave glider in head waves. With $0.17 \mathrm{~m}$ high and $2 \mathrm{~s}$ long wave, the wave glider advanced $19 \mathrm{~m}$ in $76 \mathrm{~s}$ resulted in an average speed $0.25 \mathrm{~m} / \mathrm{s}$. 


\section{Methodology of numerical simulation}

\subsection{Simulation process}

The numerical simulation for the wave glider is mainly based on the CFD software FINE/Marine, in which the simulation of the surface boat in head waves coupling with the motion of the glider has been conducted. In addition, FINE/Marine has a hybrid meshing tool and can capture large displacement changes in free surface with economic computational resources. However, due to the lack of capability to perform the simulation for the passive eccentric rotation of the hydrofoils via FINE/Marine, STAR-CCM+ is introduced to conduct this type of simulation. Therefore, in this paper, FINE/Marine is used to simulate the system response of the wave glider; and then FINE/Marine outputs its motion response to STAR-CCM+ as an input for the computation of the passive eccentric rotation of the hydrofoils; STAR-CCM+ exports the rotational motion of the hydrofoils back to FINE/Marine to simulate the system motions to perform this manual iteration until the difference in the motion response of the wave glider is less than $10^{-3}$, as shown in Fig.6.

\subsection{Governing equations}

The flow around the wave glider is modeled under isothermal conditions using the incompressible, mass and momentum equations:

$$
\begin{gathered}
\frac{\partial}{\partial \mathrm{t}} \int_{V} \rho d V+\int_{S} \rho\left(U-U_{d}\right) \cdot n d S=0 \\
\frac{\partial}{\partial \mathrm{t}} \int_{V} \rho U_{i} d V+\int_{S} \rho U_{i}\left(U-U_{d}\right) \cdot n d S=\int_{S}\left(\tau_{i j} I_{j}-p I_{i}\right) \cdot n d S+\int_{V} \rho g_{i} d V
\end{gathered}
$$

Where t is the time; $\rho$ is the fluid density, $V$ is the domain of interest, $U_{i}$ is the time averaged velocity components in Cartesian coordinates $(\mathrm{i}=1,2,3), S$ is the closed surface, $U_{d}$ is the velocity with a unit normal vector $\mathrm{n}$ directed outward. $U$ and $p$ represent, respectively, the velocity and pressure fields. $\tau_{i j}$ and $g_{i}$ are the components of the viscous stress tensor and the gravity vector, where $I_{j}$ is a vector which is equal to unity. $c_{i}$ is the volume fraction.

The finite volume method is employed to discretize the governing equations with the AVLSMART scheme Qiong et al. (2014). The realizable $\mathrm{k}-\omega$ (SST-Wilcox) model is chosen. The two transport model equations 
for the turbulent kinetic energy, $K$ and the specific dissipation rate of turbulence frequency, $\omega$, as:

$$
\begin{gathered}
\partial \rho \mathrm{K} / \partial \mathrm{t}+\partial\left(\rho U_{j} K-\left(\mu+\sigma^{*} \mu_{t}\right) \partial \mathrm{K} / \partial x_{j}\right) / \partial x_{j}=\tau_{t_{i j}} S_{i j}-\beta^{*} \rho \omega K \\
\partial \rho \omega / \partial \mathrm{t}+\partial\left(\rho U_{j} \omega-\left(\mu+\sigma^{*} \mu_{t}\right) \partial \omega / \partial x_{j}\right) / \partial x_{j}=\alpha \omega / K \tau_{t_{i j}} S_{i j}-\beta \rho \omega^{2}
\end{gathered}
$$

Here $\mu$ is the viscous coefficient. $\alpha, \beta, \beta^{*}, \sigma, \sigma^{*}$ are the turbulence model constants.

\subsection{Parameter definition}

The power of the wave glider is divided into the wave power absorption and mechanical conversion power (Liu et al. (2016), defined as:

$$
\begin{gathered}
\mathrm{P}_{W G i}=\rho g H^{2} S_{f} / 8 \mathrm{~T} \\
\mathrm{P}_{W G m}=\left(\int_{0}^{T} Z_{W G}(t) d \zeta(t) / d t d t\right) / T \\
F_{W G}=\int_{0}^{T} X_{W G}(t) d t / T
\end{gathered}
$$

Here $P_{W G i}$ and $P_{W G m}$ is the wave energy absorption power and the mechanical conversion power. $\mathrm{H}$ is the wave height. $T$ is the wave period. $S_{f}$ is waterplane area of the surface boat. $X_{W G}(t), Z_{W G}(t)$ is the surface boat time-varying forces in the horizontal and vertical directions, respectively. $\zeta(t)$ represents the heave motion. $F_{W G}$ is the time-averaged value of $X_{W G}(t)$.

The wave absorption power coefficient $\left(C_{W G P i}\right)$, the mechanical conversion power coefficient ( $C_{W G P m}$ ) of the wave glider and the thrust coefficient $\left(C_{W G T}\right)$ of the wave glider have been defined as

$$
\begin{gathered}
C_{W G P i}=P_{W G i} / 0.5 \rho C_{h y} S_{f} \dot{\xi}^{3} \\
C_{W G P m}=P_{W G m} / 0.5 \rho C_{h y} S_{f} \dot{\xi}^{3} \\
C_{W G T}=F_{W G} / 0.5 \rho C_{h y} S_{f} \dot{\xi}^{2}
\end{gathered}
$$

Where $C_{h y}$ and $S_{h y}$ are the chord and span of the hydrofoil, respectively. The propulsive efficiency of the 
wave glider, $\eta_{W G}$, is defined to the ratio of useful power over input power, as

$$
\eta_{W G}=F_{W G} \xi / P_{W G m}=C_{W G T} / C_{W G P m}
$$

The tether that connects the surface boat and the glider is seen as a spring, the force of the tether $F_{T L}$ defined as:

$$
F_{T L}=K_{T} \Delta L_{S}+T_{\text {initial }}
$$

Where $K_{T}$ stands for the stiffness, $\Delta L_{S}$ is the variation of the length $\left(\Delta L_{S} \geq 0\right)$ and $T_{\text {initial }}$ is the initial tension of the tether.

Passive induced pitching motion of the hydrofoil is governed by a linear spring acting beneath each hydrofoil according Hooke's law (see Fig.2):

$$
I_{h y} d^{2} \theta(t) / d t^{2}+T_{S} \sin (\theta(t)+\beta) d_{S}=Q(t)
$$

Where $I_{h y}$ is the inertia moment of hydrofoil, $\quad \beta$ and $d_{S}$ is constant at $7.9^{\circ}$ and $0.04 \mathrm{~m}, Q(t)$ is the resultant torque on the hydrofoil leading edge. $T_{S}$ is the linear spring force, as defined:

$$
T_{S}=k r_{S}
$$

Where $\mathrm{k}$ is the linear spring stiffness, $r_{S}$ is spring compression.

$$
\begin{gathered}
\mathrm{P}_{h y m}=\frac{1}{T}\left(\int_{0}^{T} Z_{h y}(t) d \zeta(t) / d t d t+\int_{0}^{T} Q(t) d \theta(t) / d t d t\right) \\
F_{h y}=\int_{0}^{T} X_{h y}(t) d t / T
\end{gathered}
$$

Where $\mathrm{P}_{h y m}$ is the hydrofoil mechanical conversion power, $X_{h y}(t), Z_{h y}(t)$ is the hydrofoil time-varying forces in the horizontal and vertical directions, respectively. $\theta(t)$ represents the angular (pitch) response of the hydrofoil. $F_{h y}$ is the average value of $X_{h y}(t)$.

The mechanical conversion power coefficient $\left(C_{h y P m}\right)$ and the thrust coefficient $\left(C_{h y T}\right)$ of the hydrofoil 
have been also defined as:

$$
\begin{gathered}
C_{h y P m}=P_{h y m} / 0.5 \rho C_{h y} S_{f} \dot{\xi}^{3} \\
C_{h y T}=F_{h y} / 0.5 \rho C_{h y} S_{f} \dot{\xi}^{2}
\end{gathered}
$$

Similarly, the propulsive efficiency of the hydrofoil $\eta_{h y}$, is defined based on the ratio of the thrust power over the mechanical power, as

$$
\eta_{h y}=F_{h y} \xi / P_{h y m}=C_{h y T} / C_{h y P m}
$$

\subsection{Setup in FINE/Marine}

\subsubsection{Boundary condition}

The inlet boundary is positioned $25 \mathrm{~m}$ upstream, with a pressure-outlet defined at $25 \mathrm{~m}$ downstream. The hydrostatic pressure boundary is applied to both the top and the bottom boundary conditions. The water depth is $25 \mathrm{~m}$ and the distance between the top surface and the free surface is $15 \mathrm{~m}$. The symmetry plane is applied to the transversal mid plane. And the far field boundary condition is defined to side plane $15 \mathrm{~m}$ away from the symmetry plane. Fig.7 shows the domain size and boundary conditions of the numerical simulations. A time-step size of $0.01 \mathrm{~s}$ is applied. The tether in FINE/Marine is modelled numerically as a linear spring but only with tension force and no compression force will be provided when the tether gets slack. The tether stiffness is constant, $50 \mathrm{kN} / \mathrm{m}$.

\subsubsection{Mesh generation}

The grid generation of the wave glider is conducted by use of the meshing software HEXPRESS within FINE/Marine. The meshing software HEXPRESS with the unstructured cut-cell mesh is used to generate the wave glider grid with additional refinement added adjacent to the free surface. The free surface is refined using BRICS discretization scheme which could reduce the numerical diffusion of functions adjacent to the free surface. Sliding mesh is applied to the hydrofoil rotation. Fig.8 shows the surface grid of the half surface boat (a) and the half glider (b), respectively. The first mesh layer thickness is set to $0.0001 \mathrm{~m}$. The resulting fluid mesh of the wave glider consist of approximately $7 \times 10^{6}$ cells and $7.46 \times 10^{6}$ vertices. 


\subsection{Setup in STAR-CCM+}

\subsubsection{Boundary condition}

On the other hand, the simulation of the passive eccentric rotation of the hydrofoil was conducted with the boundary conditions defined in Fig.9. Uniform static pressure is applied to the outlet condition at $2 \mathrm{~m}$ downstream. The time-varying velocity vector is applied to inlet, top and bottom condition. No-slip boundary conditions are applied to the six tandem hydrofoils. The one-DOF rotating motion option within the Dynamic Fluid Body Interaction (DFBI) module is employed to simulate the eccentric passive rotation of the hydrofoils in response to the incoming flow. In addition, a linear spring (non-torsional) coupling system as shown in Fig. 2 is introduced between the hydrofoil and the glider. The linear spring is operated in STAR$\mathrm{CCM}+$ in external forces and moments and the spring constant is $2900 \mathrm{~N} / \mathrm{m}$.

\subsubsection{Mesh generation}

The automatic meshing tool is used to generate mesh for six tandem hydrofoils simulation. As shown in Fig.10, overset mesh, around the hydrofoil, is applied to simulate the passive eccentric rotation. The first mesh layer thickness is set to $0.0001 \mathrm{~m}$ resulting in $y^{+}<1$. The total mesh number of six tandem hydrofoils approximately $3.93 \times 10^{6}$ cells and $4.28 \times 10^{6}$ vertices. 


\section{Validation of numerical method}

To build the confidence in the employed simulation methodology, two validation studies have been conducted against some published results. The first validation case is the steady simulation of the airfoil. A NACA 0012 airfoil has been studied to verify the simulation software packages used in this paper, FINE/Marine and STAR-CCM+, respectively. The experimental and numerical data of the two-dimensional foils have been compared, respectively.

The numerical simulation of the NACA 0012 airfoil has been done in the same conditions of Yousefi and Saleh (2015) simulation where the uniform inflow velocity in air is set to be $7.3 \mathrm{~m} / \mathrm{s}$ and the chord length (c) is $1 \mathrm{~m}$, which makes Reynolds number $5 \times 10^{5}$. To check the grid independence of the results, three grids $\ln$ FINE/Marine and STAR-CCM+ with an increased number of cells namely Grid 1, Grid 2 and Grid 3 are used; the results of angle of attack of $10^{\circ}$ and $16^{\circ}$ are listed in Table 2. According to Table 2(a), the differences between Grid 1 and 2 and between Grid 2 and 3 were less than 1\%. Regarding the validation in STAR-CCM+, Table 2(b) also list three grids with an increased quantity of cells namely Grid 1, Grid 2 and Grid 3. There is rarely any change in results for increasing total number of cells from 81,885 to 145,308 . To maintain gridresolution consistency for different cases and also to balance the accuracy and the computational cost, the moderate grid of Grid 2 in FINE/Marine and STAR-CCM+ is adopted for the following computation, respectively.

As shown in Fig.11, the computation results of FINE/Marine and STAR-CCM+ agreed well with the numerical simulation values. The lift and drag coefficient are consistent with the experimental data and linearly numerical results before 12 degrees. However, the stall in the numerical simulations occurred at an angle of attack of $14^{\circ}$, whereas the experimental measurements indicated that NACA0012 airfoil stalls at an angle of attack of $12^{\circ}$. The error between numerical simulation and experimental tests especially in the prediction of the stall angle is highly attributed to the imperfectly represented experimental conditions, like the Reynolds number, the incoming turbulence and the natural boundary layer caused by the wind tunnel environment, the surface roughness on the tested foil, and etc., which would all contribute to the shift of the stall angle. It can be observed in the experiments for the NACA0012 airfoil conducted by Critzos et al. at Reynolds

number $1.8 \times 10^{6}$ with respectively rough and smooth surface treatments on the foil surface. 
Further the validation study with steady foil performance evaluation, since the present study is focusing on the passive rotation of the tandem hydrofoil in STAR-CCM+, two more validation studies were added to fully understand the performance in the evaluation of oscillating hydrofoils.

The hydrodynamic performance of the oscillating foil predicted from the URANS simulations were systematically compared with experiental data from the field tests. By using the test conditions stated in Kinsey and Dumas (2012), a two-dimensional single oscillating foil and a three-dimensional tandem oscillating foils case with configured in line are carried out. The chord based Reynolds numbers is 500,000. A rigid foil with NACA0015 cross-section shape and the chord length is $0.24 \mathrm{~m}$. The reduced frequency is equal to 0.14 . The aspect ratio of the three-dimensional foil is 7 . The heave amplitude and the pitch amplitude are equal to the chord length and $75^{\circ}$ respectively. The distance between the upstream foil and downstream foil is $1.296 \mathrm{~m}$. The oscillating phase difference in tandem case between the two foils is $180^{\circ}$. According to the mesh criteria of Kinsey and Dumas (2012) , the nodes on foil profile is 500 and inter-foil resolution is $0.024 \mathrm{~m}$ is applied for validation.

The time histories of an oscillating foil's thrust coefficient for both present and published results are shown in Fig.12 (a). There is a good agreement between the results of an oscillating foil and the published results of Kinsey and Dumas (2012). From the result, it is clear that the loss of thrust approximately $12 \%$ for the three-dimensional hydrofoil compared to the two-dimensional hydrofoil. However, the two tandem hydrofoil thrust has been improved. The average thrust coefficient of the tandem oscillating foils over one period also compare well with the published results of Kinsey and Dumas (2012) in Table 3, and the difference between present simulations and the published results is less than $3 \%$. Furthermore, the difference is thrust between two-dimensional and three-dimensional hydrofoil is less than $10 \%$. It implies that the hydrofoils configuration in tandem can reduce the loss of thrust due to three-dimensional effects. 


\section{Results and discussion}

\subsection{The wave glider under the regular wave in FINE/Marine}

The numerical simulation of the wave glider consists of eight and a half wave periods where the motion tends to stabilize after two wave periods. Velocity residuals in heave and surge mode reach full convergence, meeting the requirements of the program iteration, as shown in Fig.13.

There is no significant difference in heave response between the surface boat and the wave glider in Fig.14 (a). The surface boat heave velocity is consistent with that of the glider in Fig.14 (b). It is equally obvious to be observed from Fig.15 that the wave glider gradually reaches a steady state after two wave periods and move forward periodically in the fourth wave period. Additionally, the horizontal speed of the surface boat and the glider rises alternately, which means that the glider constantly tugs the surface boat and moves in a pulse form. For the surface boat horizontal velocity, it continues to accelerate from crest to trough and then drop until it reaches the crest again which is attributed to the surge force on the surface boat. According to Fig.15, via averaging the surface boat's horizontal velocity over the experienced time, the average velocity of the wave glider is $0.259 \mathrm{~m} / \mathrm{s}$. Compared with the tank experiments, the average horizontal velocity error of the wave glider is $3.6 \%$. It can be seen that the horizontal force of the surface boat changes periodically after the second wave period in Fig.16. The wave energy absorption power of the wave glider is $9.869 \mathrm{~W}$ and the average thrust force of the surface boat is $4.403 \mathrm{~N}$ based on Eq. (5) and Eq. (7), respectively. Therefore, the thrust efficiency of the wave glider is $11.556 \%$.

The tether in FINE/Marine is modelled numerically. The tether of the wave glider also moves in a pulse form in Fig.17 (a). It reveals that the tether is always in tension and the vertical force tends to be stabilizing earlier than the horizontal one. Moreover, the maximum oscillating angle is less than $2^{\circ}$.

\subsection{The propulsion efficiency of the passive eccentric rotation of the hydrofoils in STAR-CCM+}

In the simulation of the passive eccentric rotation, there are six tandem hydrofoils, namely hydrofoil-1, hydrofoil-2, hydrofoil-3, hydrofoil-4, hydrofoil-5, hydrofoil-6 from stem to stern. The distance between adjacent hydrofoils is $0.09 \mathrm{~m}$. The distance between the passive eccentric rotation axis of each hydrofoil to its leading edge is $0.05 \mathrm{~m}$. 
Among the hydrofoils, except for the hydrofoil-2, the average thrust force of remaining hydrofoils is positive as shown in Table 4. Moreover, hydrofoil-4 has the maximum value while hydrofoil-3 and hydrofoil-5 thrust forces are similar. For the average mechanical conversion power, hydrofoil-5 acquires the largest mechanical conversion power, followed by hydrofoil-4. There is no significance change between hydrofoil- 4 and hydrofoil-3. However, hydrofoil-1 and hydrofoil-2 offer lower conversion. This may be due to the difference in torque of each hydrofoil, since the hydrofoils are fixed to the glider and they have the same movement according to the Eq. (15).

Fig.18 summarizes the thrust efficiency of the hydrofoils computed from Table 4 . Although the thrust force of hydrofoil- 1 is not the largest, but it requires the least mechanical conversion power. Thus the thrust efficiency of hydrofoil-1 is the most effective. Hydrofoil- 4 , hydrofoil- 5 and hydrodfoil- 3 are sequentially reduced. The thrust efficiency of hydrofoil- 2 is negative since the average thrust force is negative.

Based on the previous discussion, the last wave period and cross-section (parallel to the root of the hydrofoil with an offset of $0.15 \mathrm{~m}$ ) are selected for calculating propulsion efficiency of the passive eccentric rotation of the hydrofoils and investigating the wake interaction between the hydrofoils. The average thrust coefficient as displayed in Fig.19 reveals that the curve tends to be relatively flat although the average thrust coefficient magnitudes differ. Hydrofoil- 4 and hydrofoil- 5 have a thrust enhancement compared to a single hydrofoil (hydrofoil-1). The average thrust coefficient of hydrofoil-3 is slightly lower than or close to that of hydrofoil-1. Hydrofoil-6, situated at the end of tandem hydrofoils, is the least contributor among the hydrofoils; while hydrofoil-2 has a negative value and plays a resistance role. Apart from the average thrust coefficient magnitude, the rear hydrofoils (from hydrofoil-2 to hydrofoil-6) have the same temporal trend. $\overline{\mathrm{C}}_{\mathrm{T}}$ Keeps growing until $\mathrm{T}=0.3$, and then it reduces at the end of the downstroke. Moreover, $\overline{\mathrm{C}}_{\mathrm{T}}$ continues to decrease between $\mathrm{T}=0.5$ and 0.6 and increase slowly until the end of the upstroke.

Fig. 20 shows the passive rotation of six tandem hydrofoils. The range of rotation angle as observed is ranging from $-30^{\circ}$ to $30^{\circ}$, indicating that the hydrofoils do not exceed the allowable flapping angle. In addition, six tandem hydrofoils rotate in clockwise when the glider rises and rotate in anti-clockwise when the glider falls. Moreover, the clockwise rotation angle is larger than the anti-clockwise rotation angle when the glider is in the trough of wave. 


\subsection{Detailed flow analysis for the hydrofoils in the passive eccentric rotation}

To find out the reason of the above performance differences, the flow structure is examined. Fig.21 displays a time series of snapshots of the vorticity contour for the six tandem hydrofoils in 8 different time stamps within one cycle ( $\mathrm{T}=0,0.175,0.3,0.4,0.5,0.66,0.8,1.0$ is marked in Fig. 19 and Fig. 20).

Fig.21 (a-e) shows the vortex structure during downstroke. As shown in the figures, as a global observation, the vortex structure around the front hydrofoil is more stable compared to the ones around the after hydrofoils which is largely affected by the shed vortex from the previous hydrofoils.

From Fig.21 (b) to Fig.21 (c) the vortices on the lower surface move towards the trailing edge, disperse away and encounter its adjacent hydrofoil, followed by a new set of leading-edge vortices (LEV) formed on the upper surface of the hydrofoils; and then they move on passing the rotation axis toward to the trailing edge, which affect the hydrofoil rotation. For example, the LEV from the hydrofoil-3 (vortex A) and the hydrofoil-6 (vortex B) begin to detach from the hydrofoils and gradually get weakened in the wake, by which the rotation angles of hydrofoil-3 and hydrofoil- 6 will be increased.

As the hydrofoil-2 continues to rotate anticlockwise to its maximum rotational angle, the LEV (vortex c) decomposes and becomes weaker on the hydrofoil trailing edge as shown in Fig.21 (d) and Fig.21 (e); while comparing the Fig.20, the residing LEVs on leading edge of the hydrofoil-1(vortex D) and the hydrofoil-4 (vortex E) provides an enhancement for them to rotate reversely. Through the comparison of the rotation angles of the hydrofoil-2 and the hydorofoil-4 (or hydrofoil-1), there is significance difference phase between the pitching motion and heave motion of the hydrofoil-2, which may be the reason why the thrust of the hydrofoil-2 is negative.

The hydrofoils will then continue to rotate clockwise and restore to the original position thanks to the contribution of the spring force (seen from Fig.21 (f) to Fig.21 (h)). The above findings suggest that the interaction of the vortices with the hydrofoils is crucial to the force generation on the hydrofoils. In addition, the application of the springs in the current design only control the hydrofoils to rotate synchronously without contributing to the increase of the thrust of the hydrofoils. In the future study the spring will be further optimized to maximize the thrust of the hydrofoils. 


\section{Conclusion}

In the previous studies, the research efforts have focused on the model experiments and lack of systematic numerical simulation of the wave glider, especially the addition of the passive eccentric rotation of the hydrofoil. Therefore, a method for the unsteady RANS simulation for the motion of the wave glider is proposed. The method can successfully be utilized to compute the flow around the wave glider and the force and moment characteristics. The surge force of the surface boat and the passive eccentric rotation of the hydrofoil are the factors that affect the propulsion efficiency of the wave glider, especially the interaction of the front hydrofoil and the rear hydrofoil. Furthermore, the results of the tank experiment and numerical simulation are found to be in excellent agreement. However, more studies are required for optimum design and simulation of propulsion efficiency of the hydrofoil in different sea conditions.

\section{Acknowledgments}

The authors would like to take this opportunity to send their special thanks to the National Ocean Technology Center for their support and financial funding for the current research [ Grant numbers: 2014AA09A507]. 


\section{Reference}

Baoqiang, T., Jiancheng, Y., Aiqun, Z., Wenming, J., Wentao, Z., Er, C.Z., 2014. Analysis On Movement Efficiency Forwave Driven Unmanned Surface Vehicle. ROBOT. (01), 43-48.

Critzos, C., Heyson, H., Boswinkle, R., 1955. Aerodynamics Characteristics of Naca0012 Airfoil Section at Angle of Attacks From 0 Degrees to 180 Degrees. Technical Report Archive \& Image Library. 79

Elhadad, A., Duan, W.Y., Deng, R., Elhanfey, H., 2014. Numerical Analysis for Resistance Calculations of Npl as a Floating Hull for Wave Glider., Bangkok, Thailand.

Jacobs, E., Sherman, A., 1937. Airfoil Section Characteristics as Affected by Variations of the Reynolds Number.

Technical Report Archive\& Image Library. 8

, pp.286-290.

Jia, L.J., Zhang, X.M., Qi, Z.F., Qin, Y.F., Sun, X.J., 2014. Hydrodynamic Analysis of Submarine of the Wave Glider.

Kinsey, T., Dumas, G., 2012a. Computational Fluid Dynamics Analysis of a Hydrokinetic Turbine Based On Oscillating Hydrofoils. Journal of Fluids Engineering, Transactions of the ASME. 134(2)

Kinsey, T., Dumas, G., 2012b. Computational Fluid Dynamics Analysis of a Hydrokinetic Turbine Based On Oscillating Hydrofoils. Journal of Fluids Engineering, Transactions of the ASME. 134(2)

Kraus, N.D., 2012. Wave Glider Dynamic Modeling, Parameter Identification and Simulation.

Liu, P., Su, Y., Liao, Y., 2016. Numerical and Experimental Studies On the Propulsion Performance of a Wave Glide Propulsor. China Ocean Eng. 30(3), 393-406.

Ngo, P., Al-Sabban, W., Thomas, J., Anderson, W., Das, J., Smith, R.N., 2013. An Analysis of Regression Models for Predicting the Speed of a Wave Glider Autonomous Surface Vehicle., Sydney, NSW, Australia.

Qiong, W., Xue-Mei, F., Li-Ping, J., Xing-Rong, S., Rong-Quan, C., 2014. Numerical Simulation of Hydrodynamic Performance of Podded

Propulsor with Numeca Software. The 13th National Symposium on Hydrodynamics and the 26th National Symposium on Hydrodynami, Qingdao, China.

Smith, R.N., Das, J., Hine, G., Anderson, W., Sukhatme, G.S., 2011. Predicting Wave Glider Speed From Environmental Measurements., Kona, HI, United states.

Tian, B., Yu, J., Zhang, A., 2015. Lagrangian Dynamic Modeling of Wave-Driven Unmanned Surface Vehicle in Three Dimensions Based On the D-H Approach., 390, Qingnian Street, Heping District, Shenyang, China.

Tian, B., Yu, J., Zhang, A., Zhang, F., Chen, Z., Sun, K., 2014. Dynamics Analysis of Wave-Driven Unmanned Surface Vehicle in Longitudinal Profile., Taipei, Taiwan.

Yousefi, K., Saleh, R., 2015. Three-Dimensional Suction Flow Control and Suction Jet Length Optimization of Naca 0012 Wing. Meccanica. 50(6), 1481-1494.

Zheng, B.H., Xu, C.Y., Yao, C.L., Chen, J.W., 2015. The Effect of Attack Angle On the Performance of Wave Glider Wings. AMM. 727-728, 587-591. 


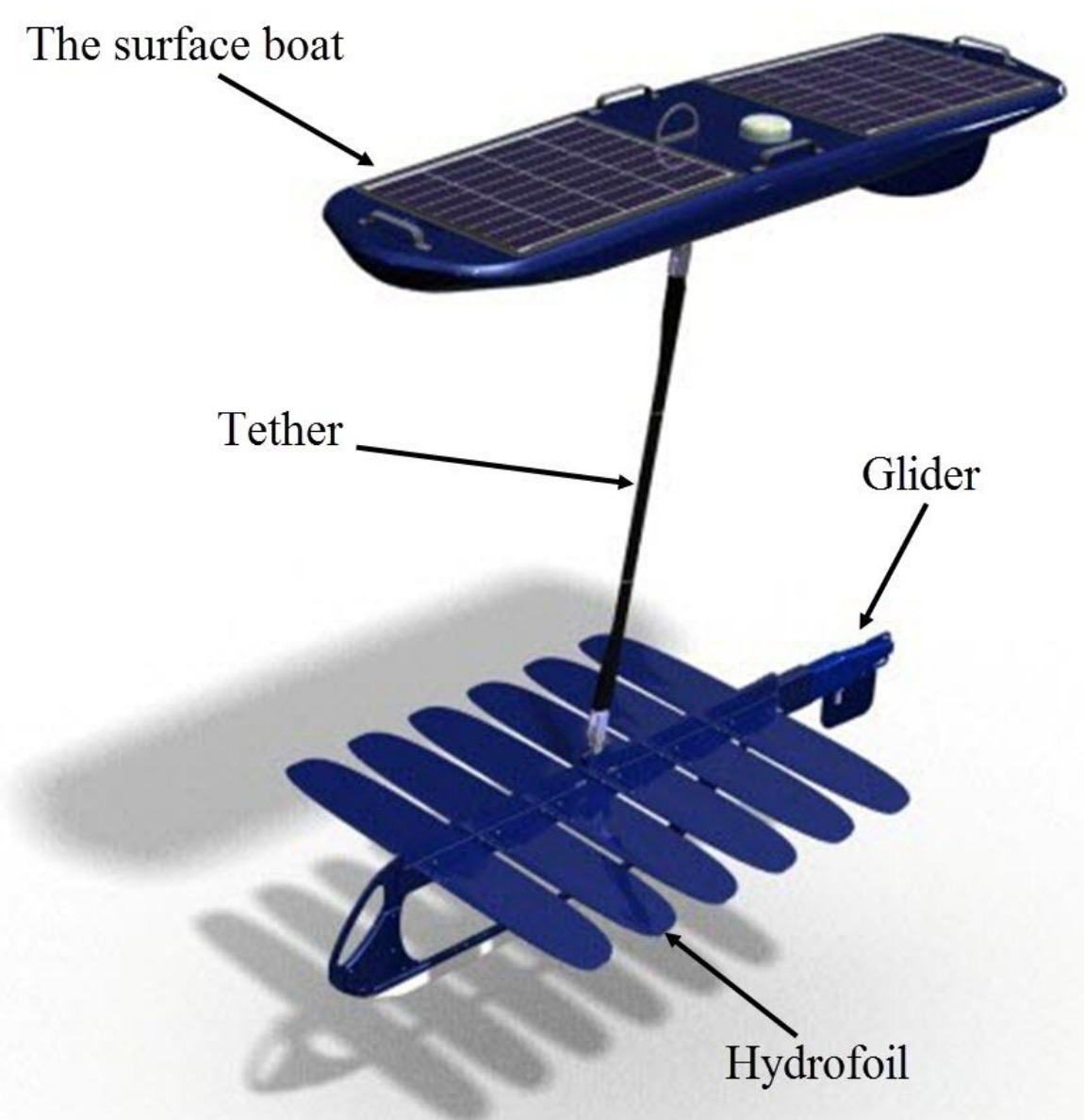

Fig.1. The wave glider developed by China National Marine Technology Center

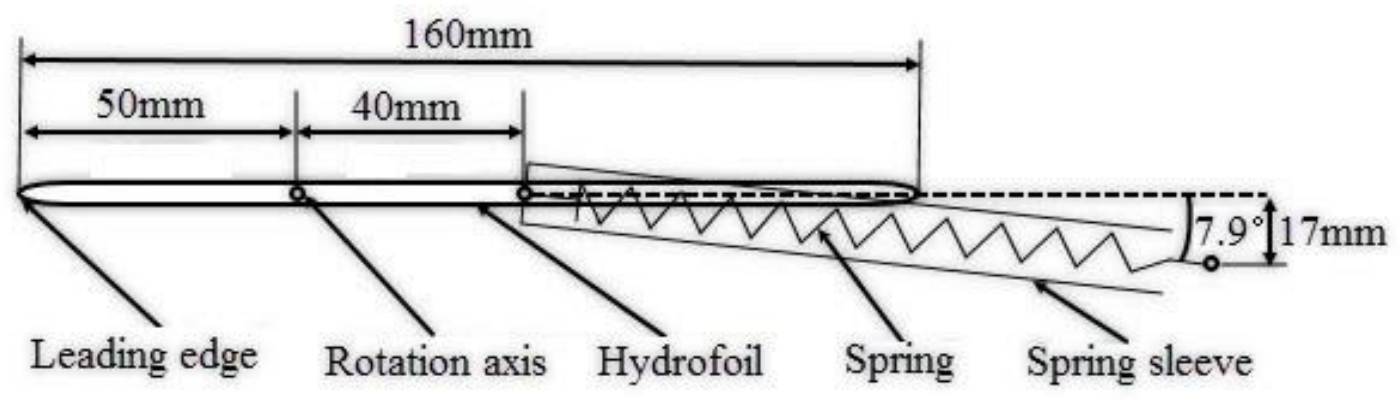

Fig.2. The spring installation diagram 


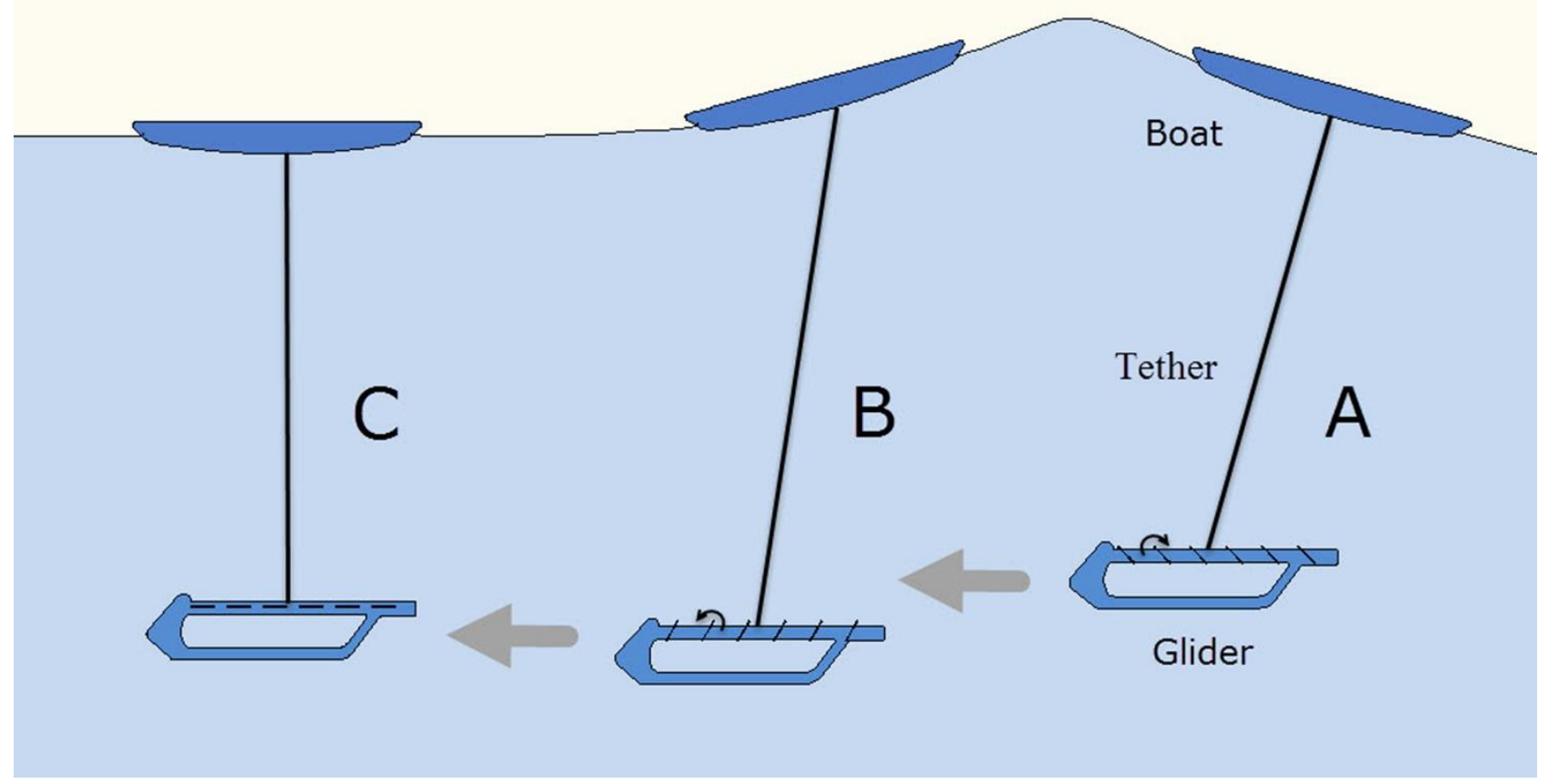

Fig.3. Operational mechanism of wave glider 


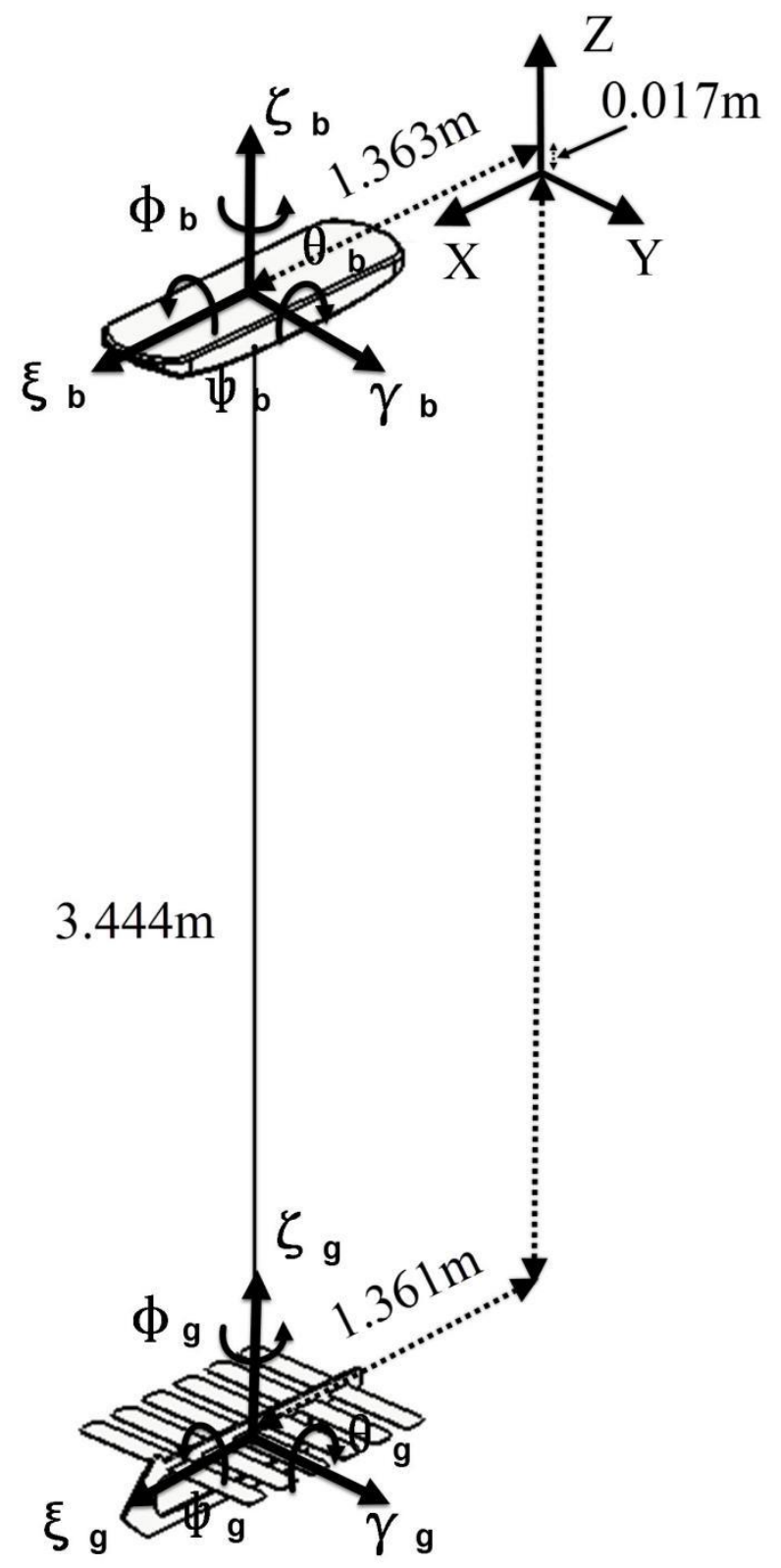

Fig.4. Coordinate system of wave glider 


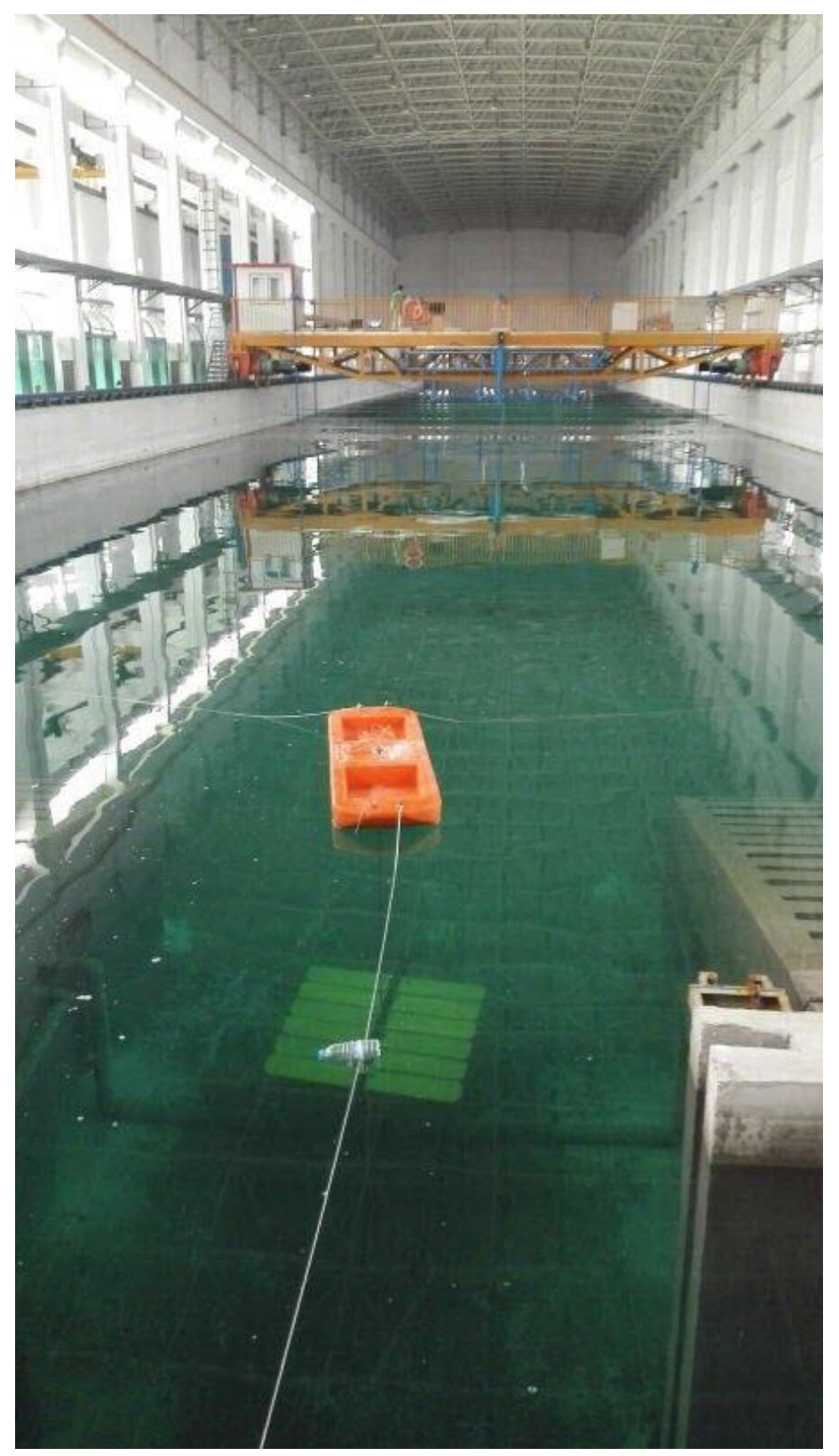

Fig.5. Free sailing test of the wave glider in waves 


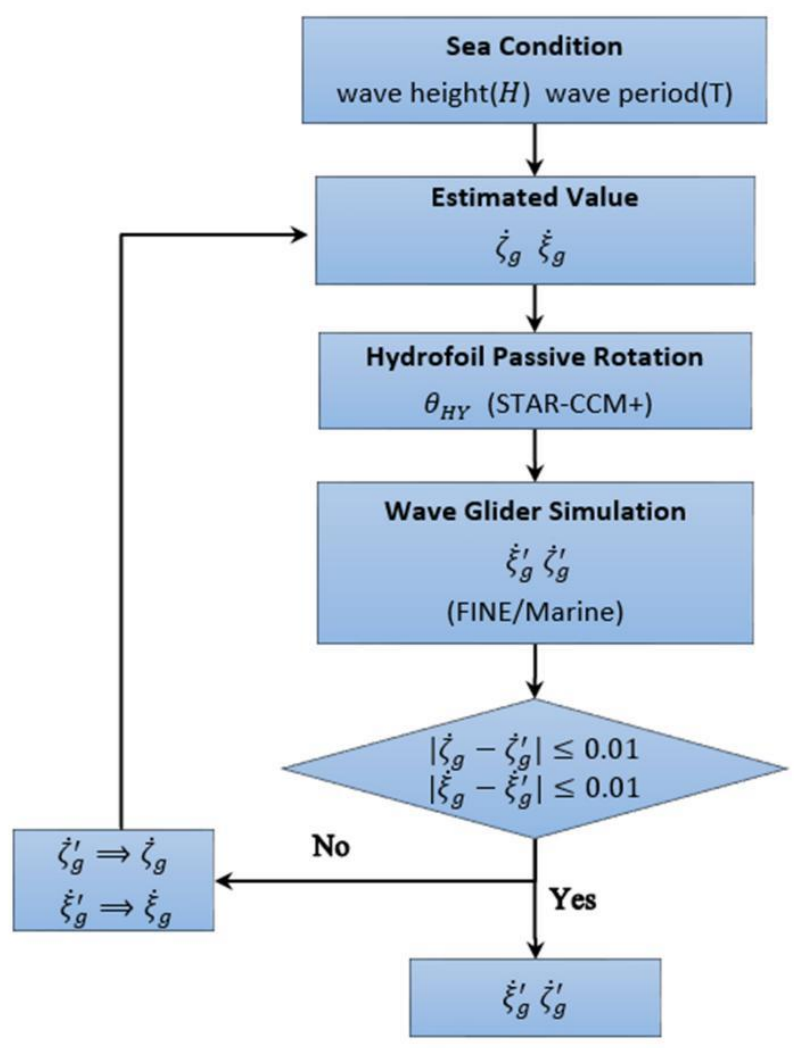

Fig.6. Schematic diagram of CFD simulation 


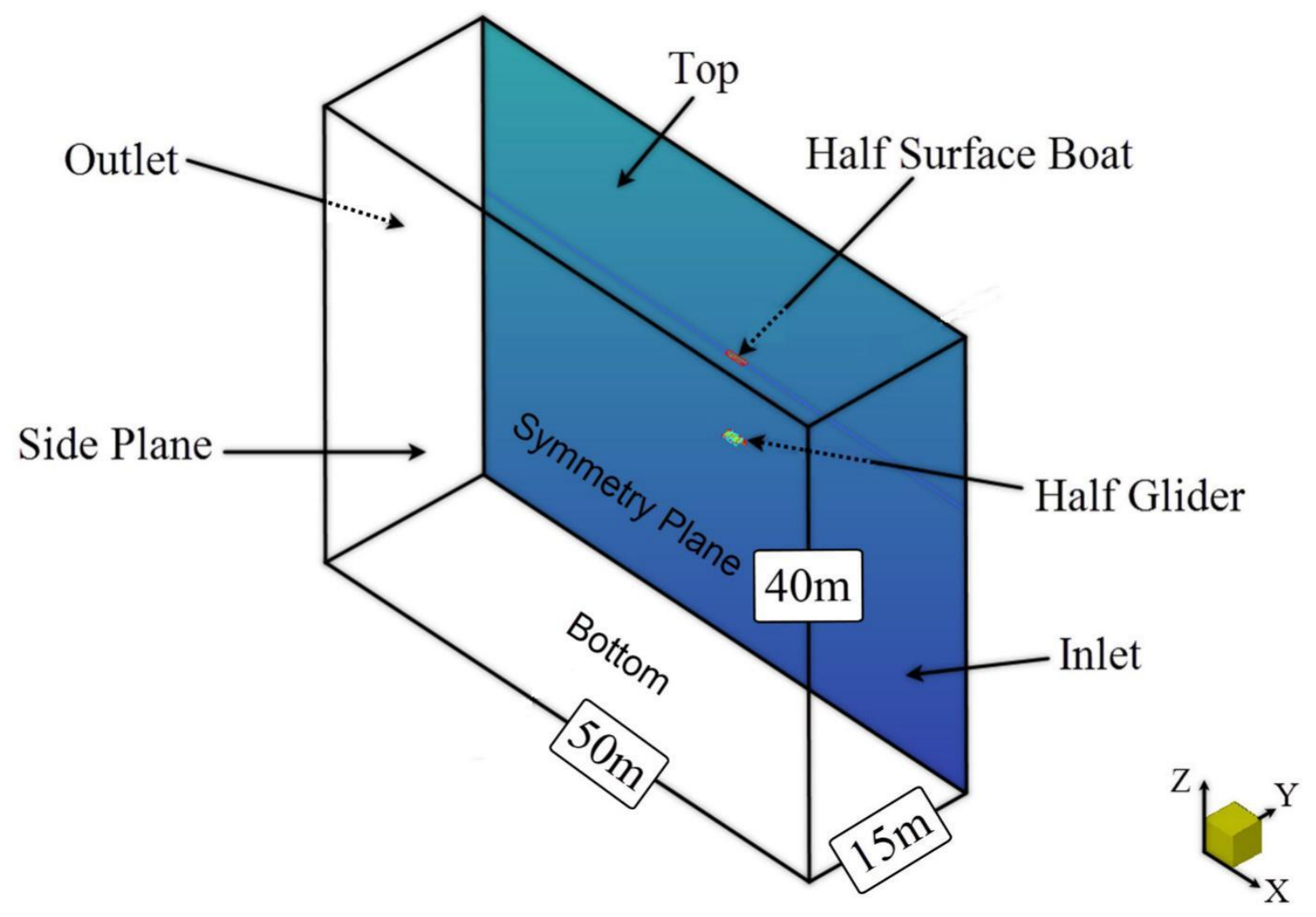

Fig.7. Domain size and boundary conditions for numerical simulations of the wave glider in FINE/Marine

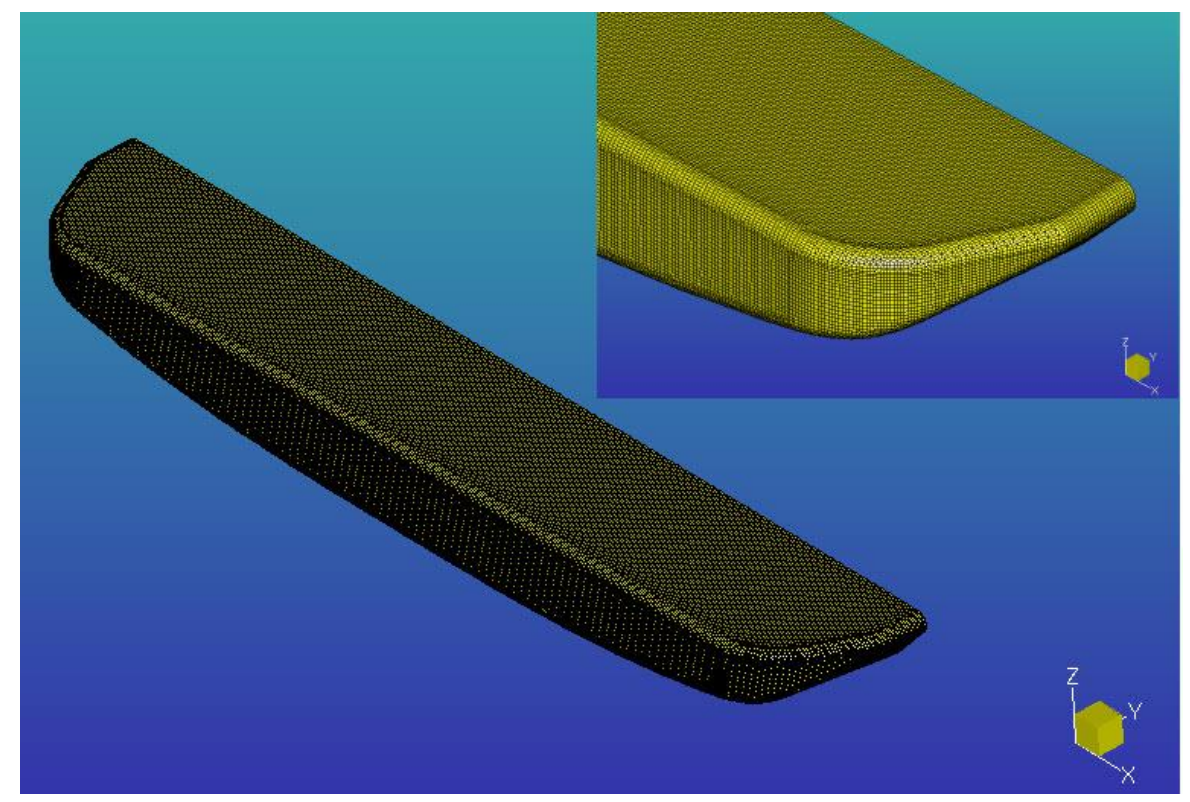

Fig.8 (a). Surface grid of the half surface boat 


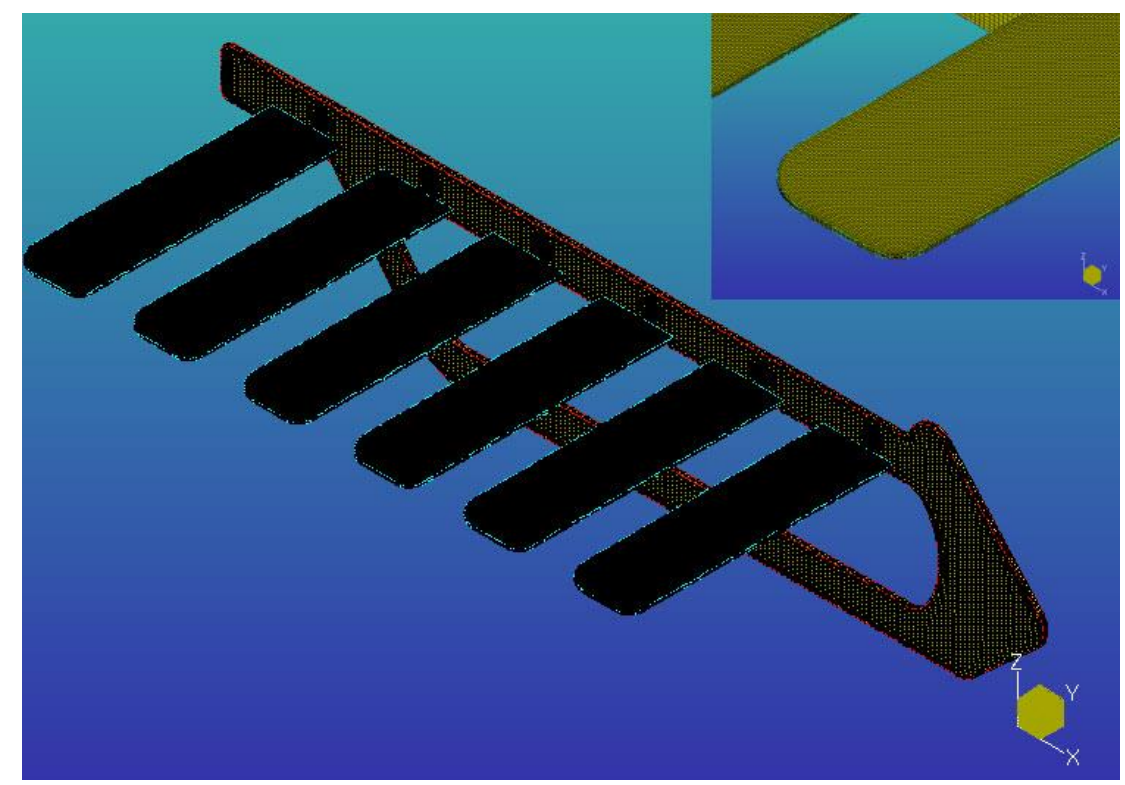

Fig.8 (b). Surface grid of the half glider

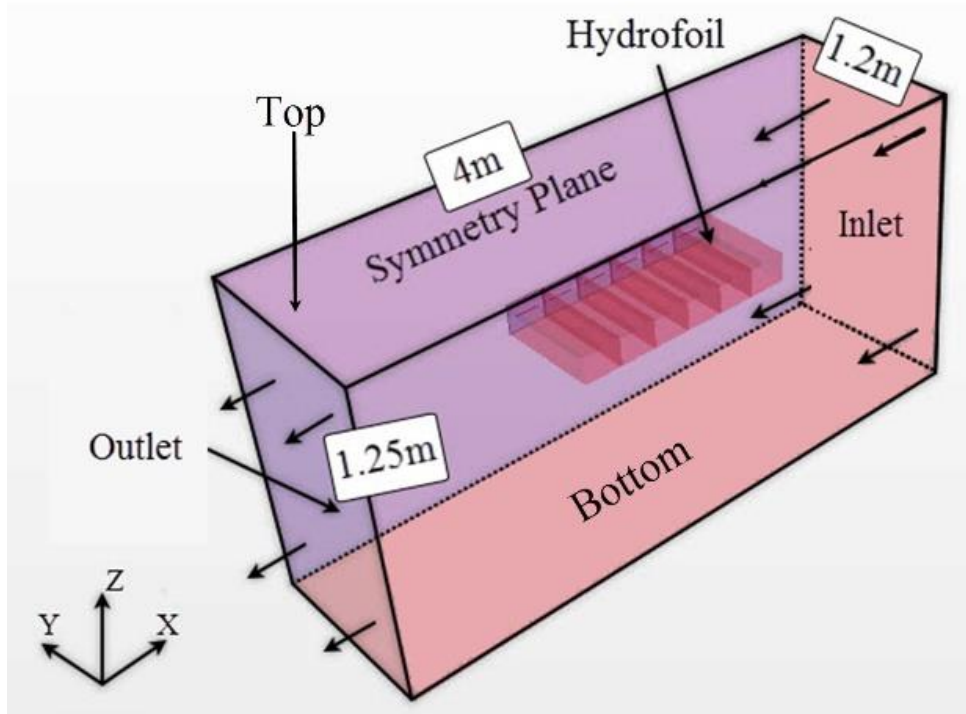

Fig.9. Domain size and boundary condition for the passive eccentric rotation of the hydrofoils 


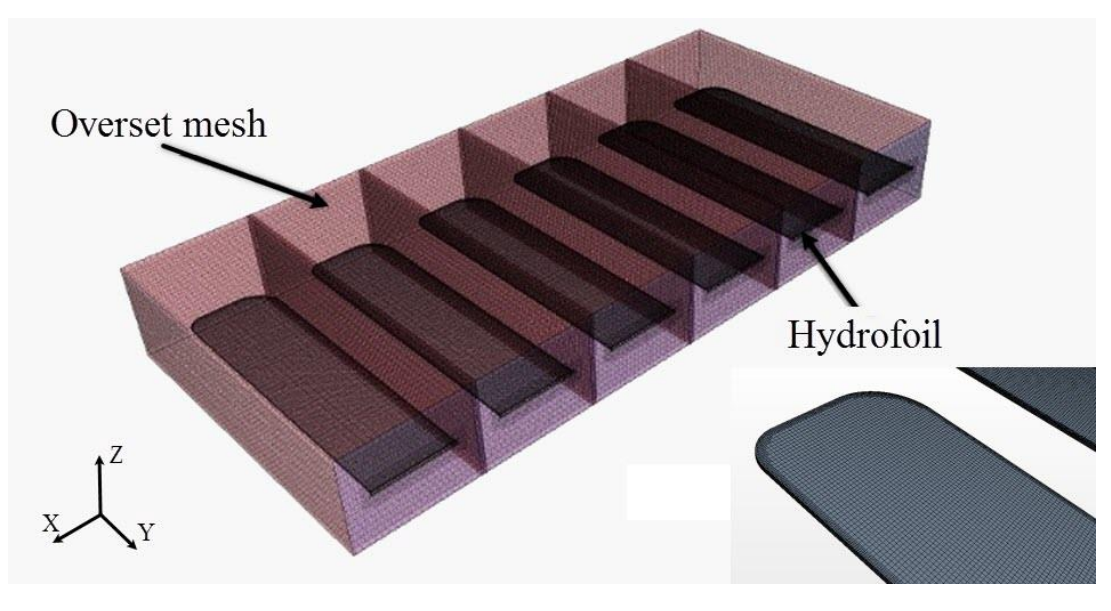

Fig.10. The close-up view of six tandem hydrofoils grid

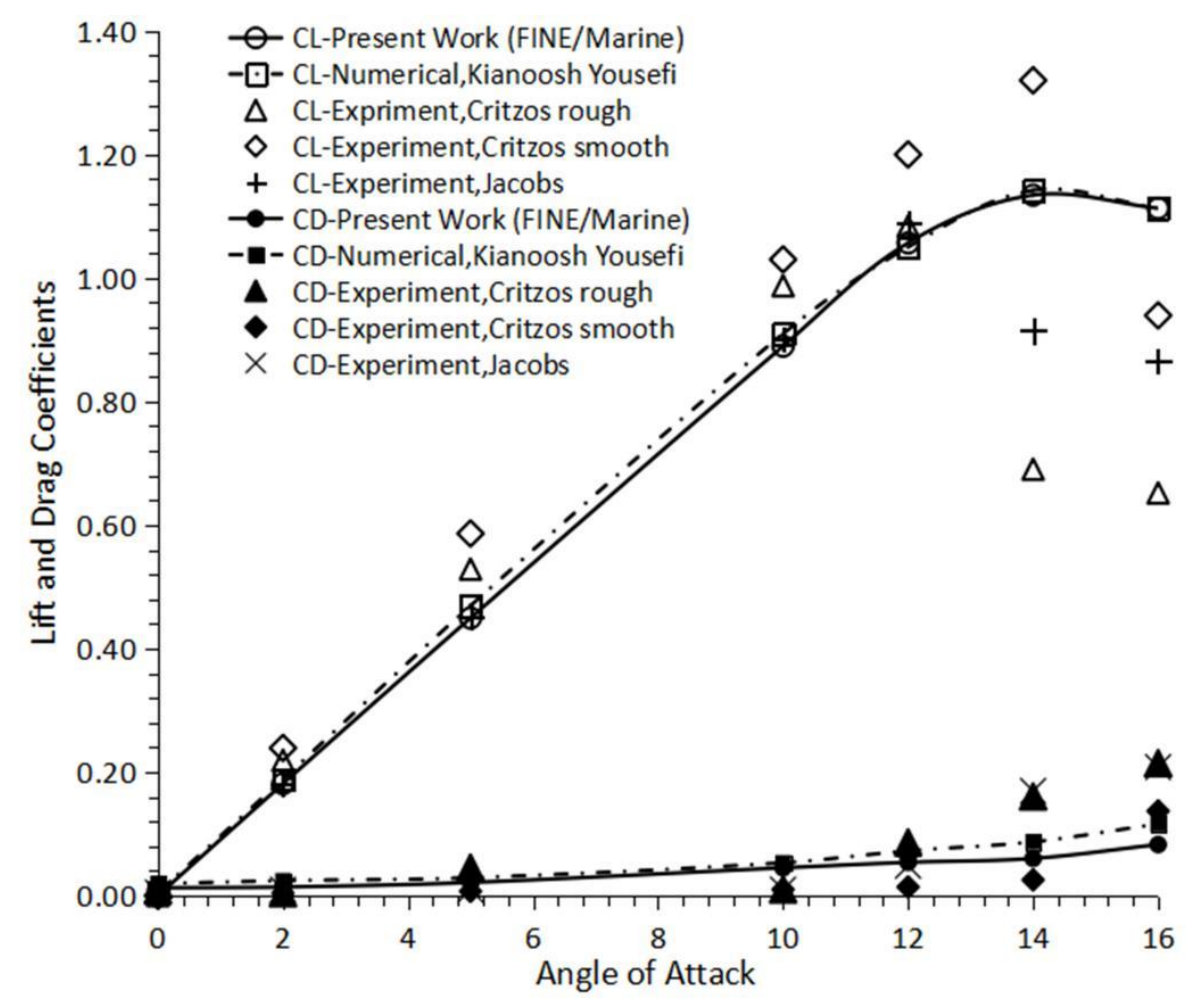

Fig.11 (a). Comparison in FINE/Marine among the current computational results, the numerical work results of Yousefi and Saleh (2015) and the experimental results of Jacobs and Sherman (1937) and Critzos et al. 


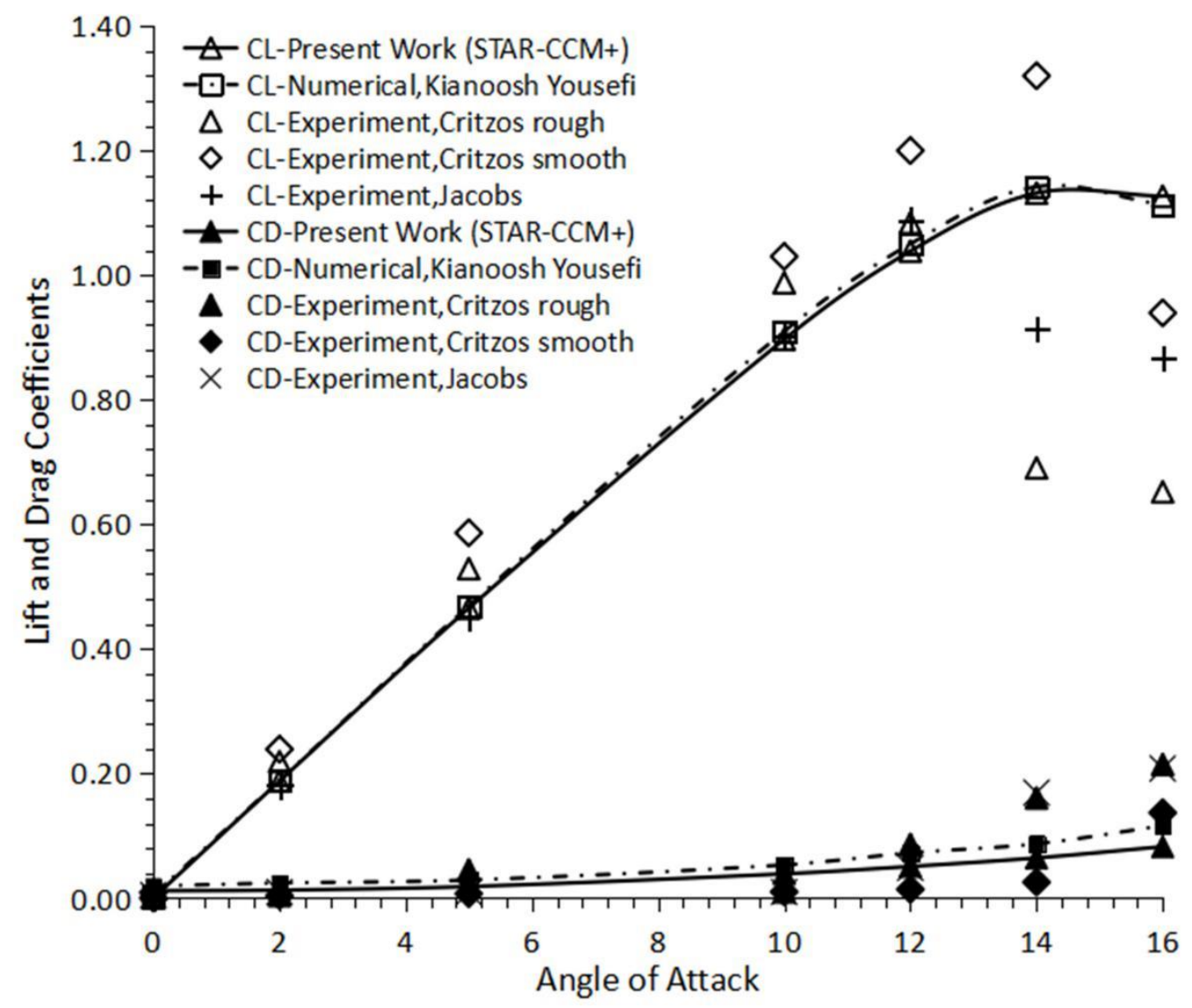

Fig.11 (b). Comparison among the current computational results, the numerical work results of Yousefi and Saleh (2015) and the experimental results of Jacobs and Sherman (1937) and Critzos et al. (1955)

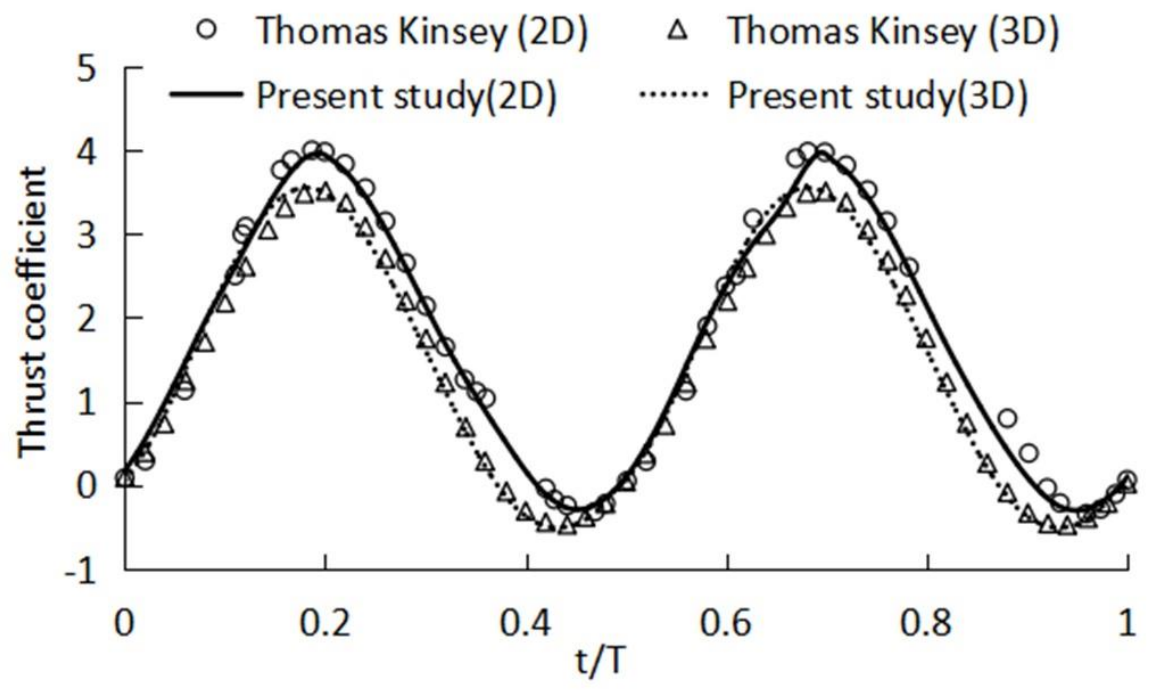

Fig.12. Validation results on thrust coefficient for an oscillating foil in STAR-CCM+ which compared with Kinsey and Dumas (2012) 


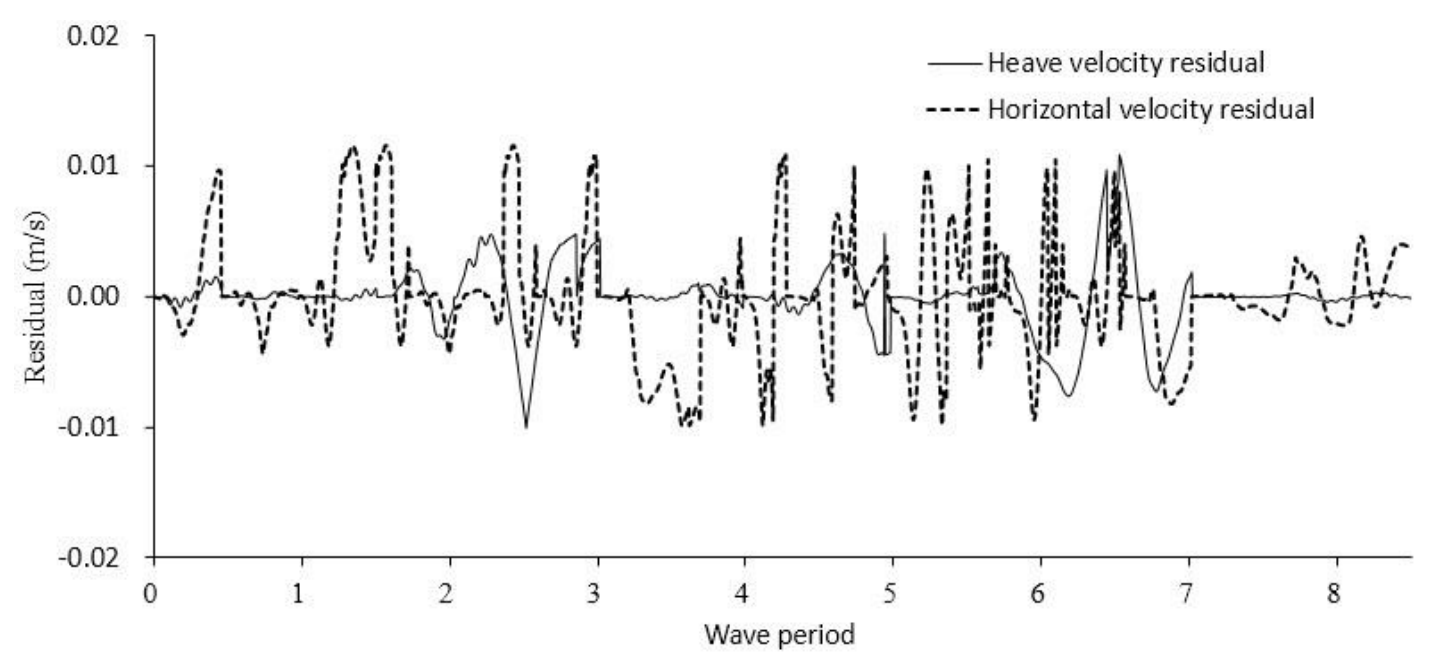

Fig.13. The convergence heave and horizontal velocity residuals of the wave glider

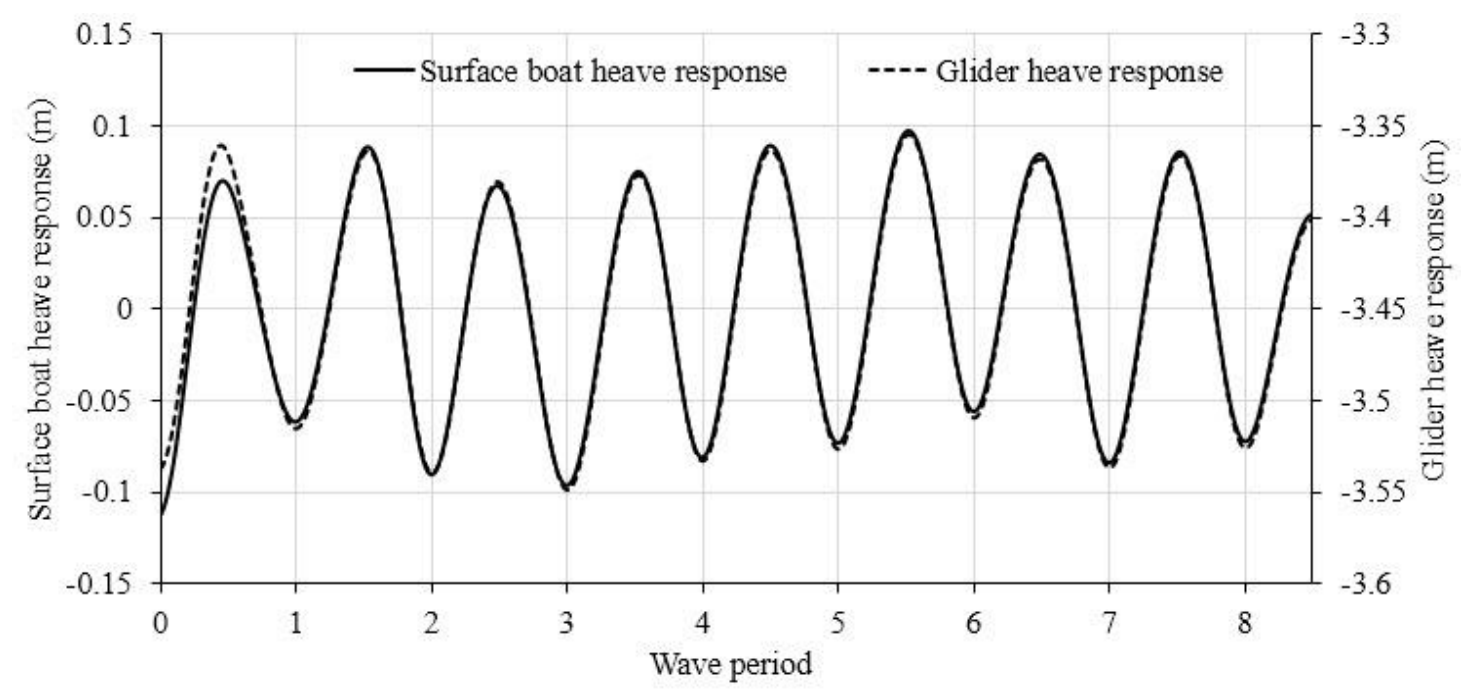

Fig.14 (a). The wave glider heave response

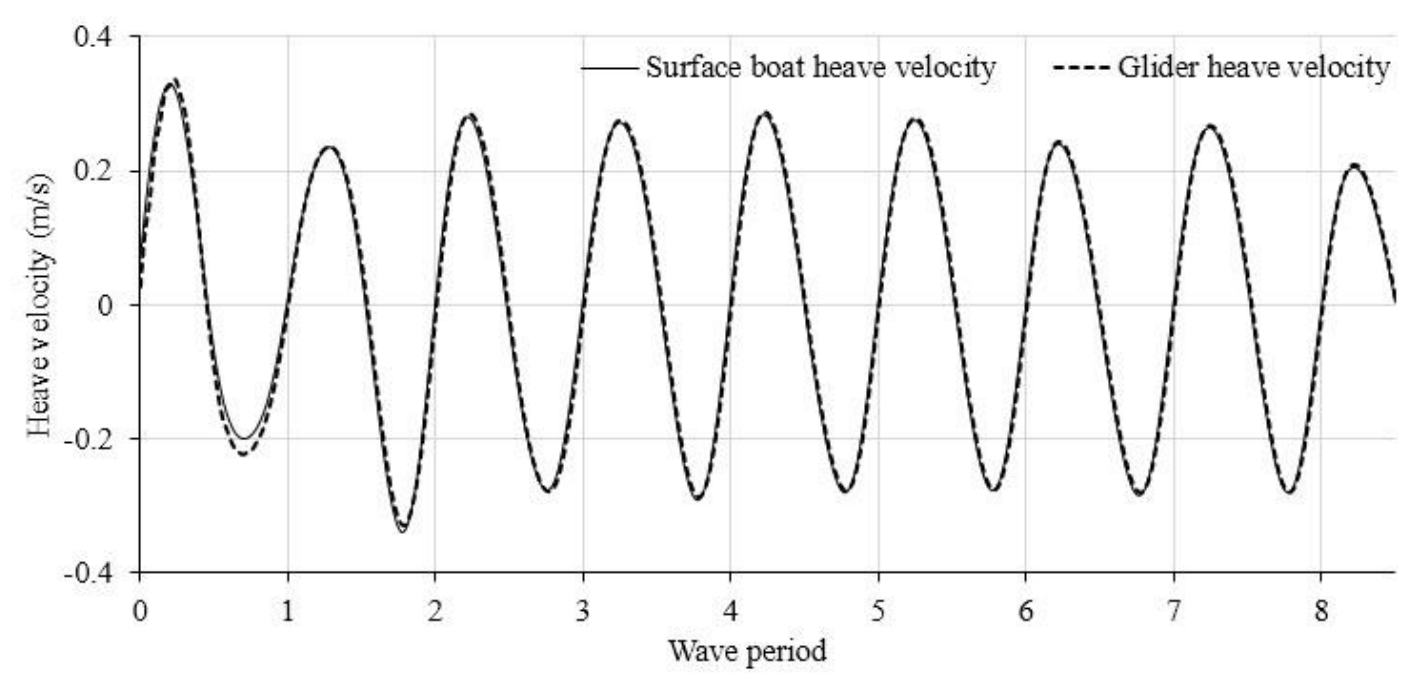


Fig.14 (b). The wave glider heave velocity

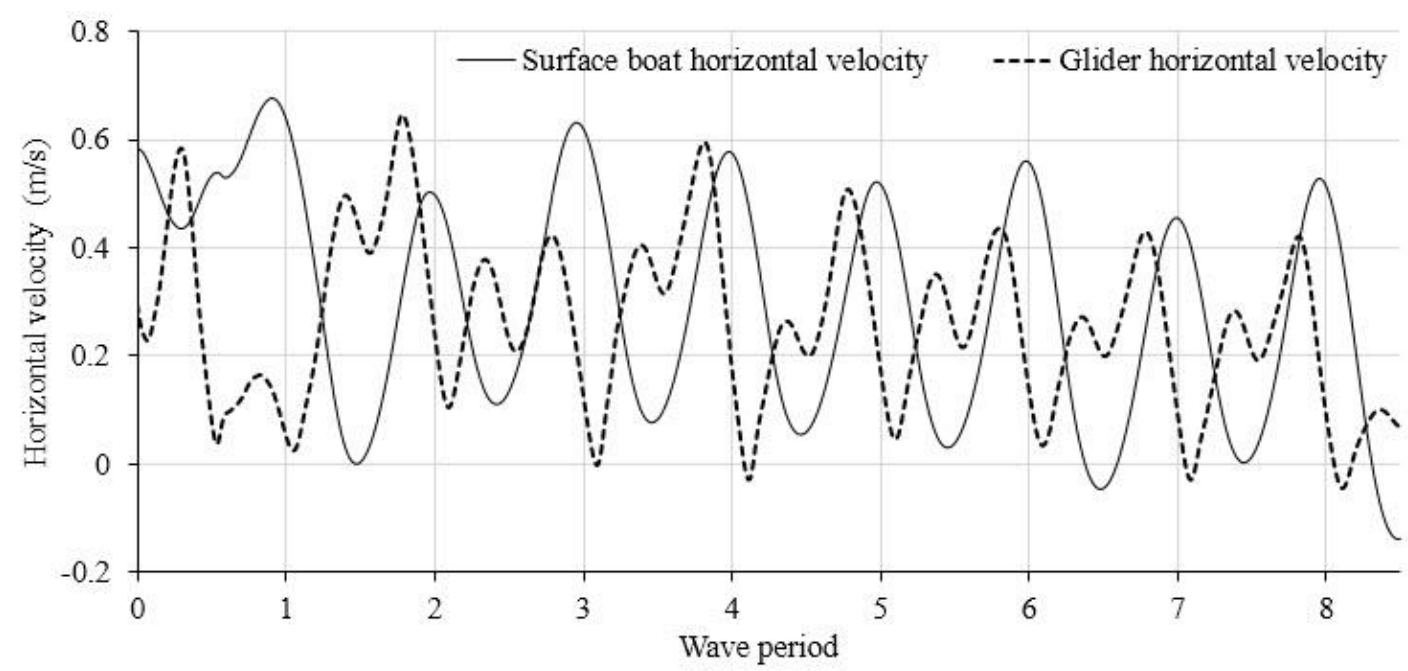

Fig.15. The wave glider horizontal velocity

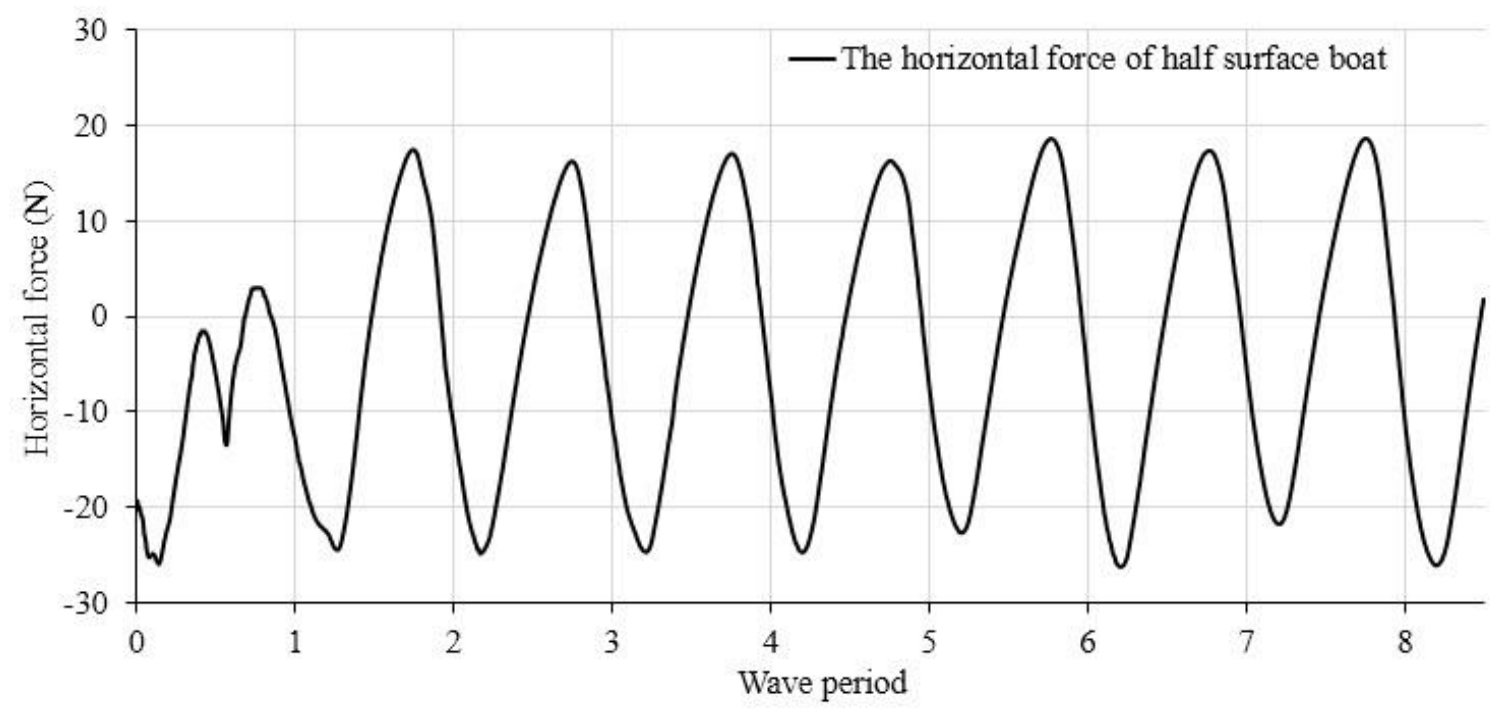

Fig.16. The horizontal force of the surface boat 


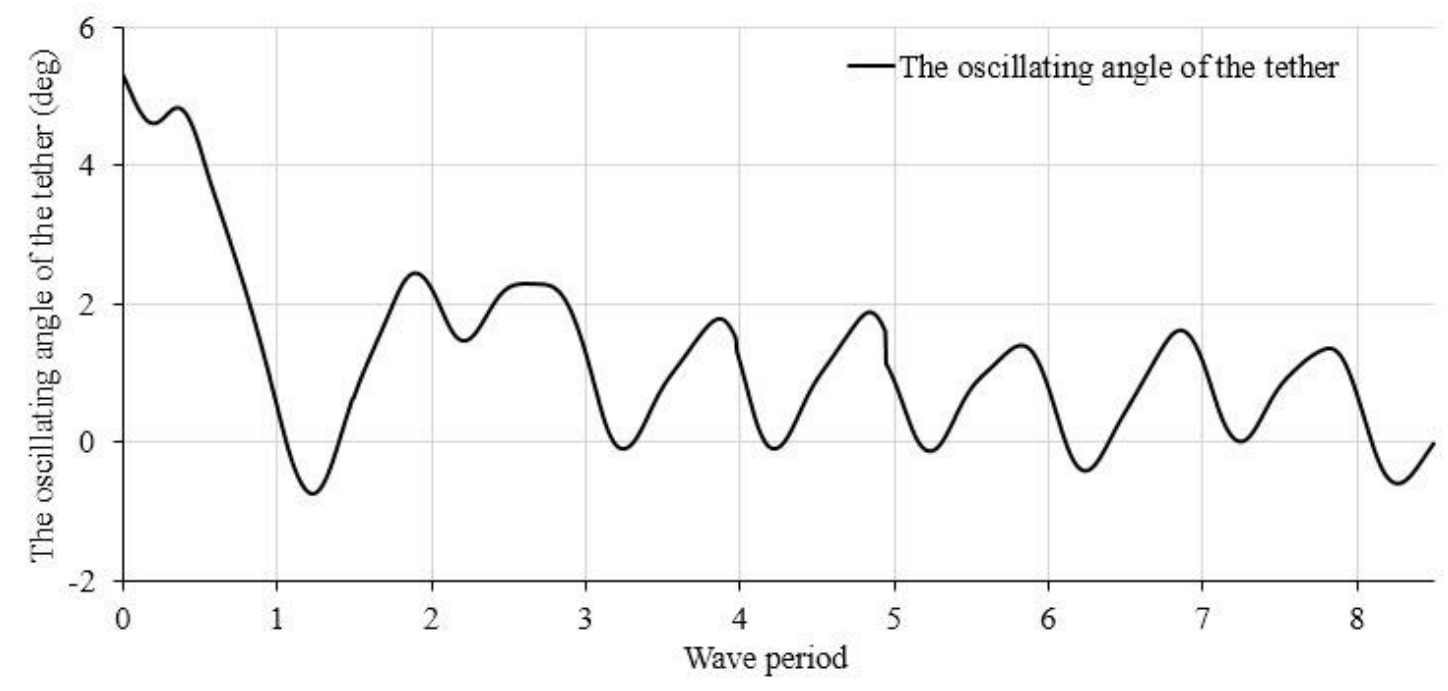

Fig.17 (a). The oscillating angle of the tether

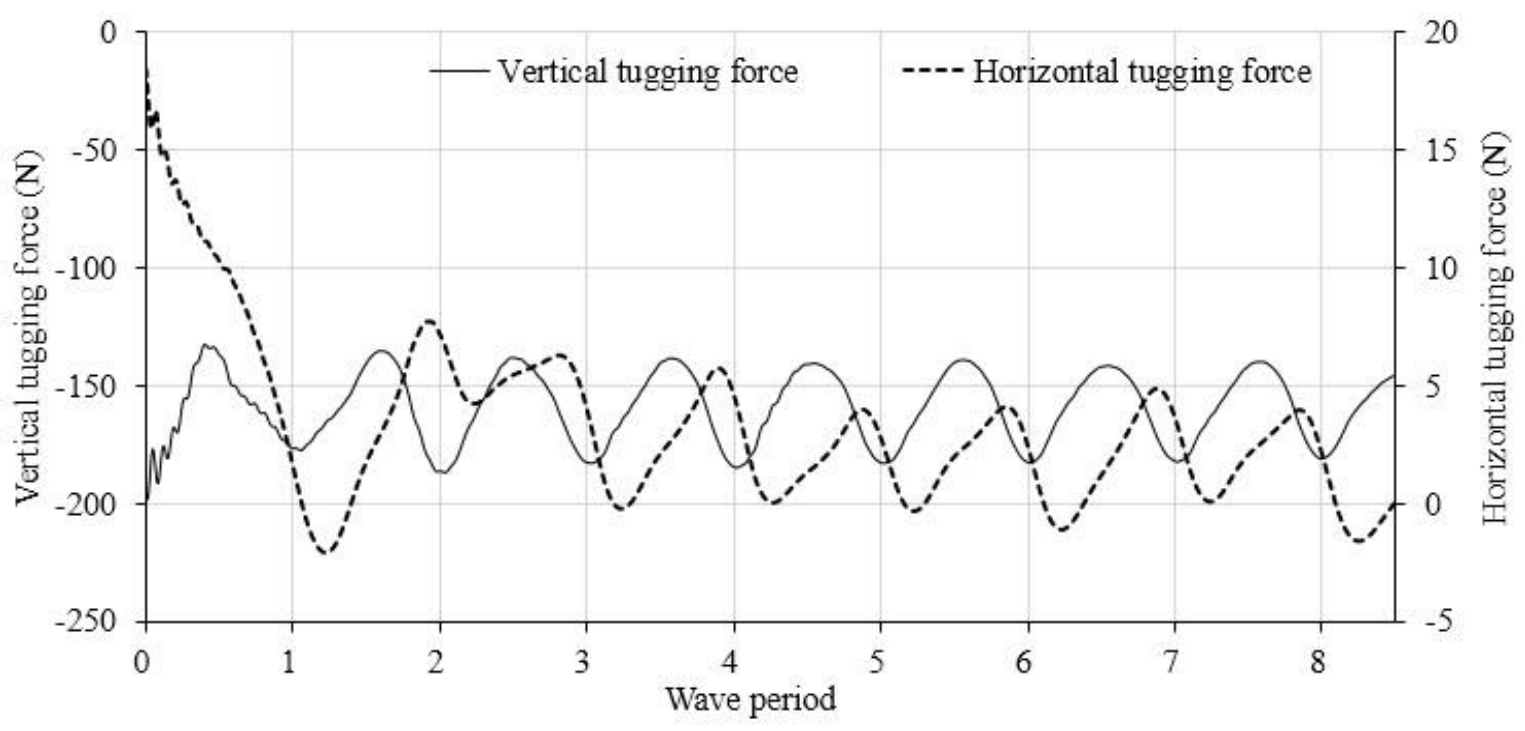

Fig.17 (b). The tugging force of the tether 


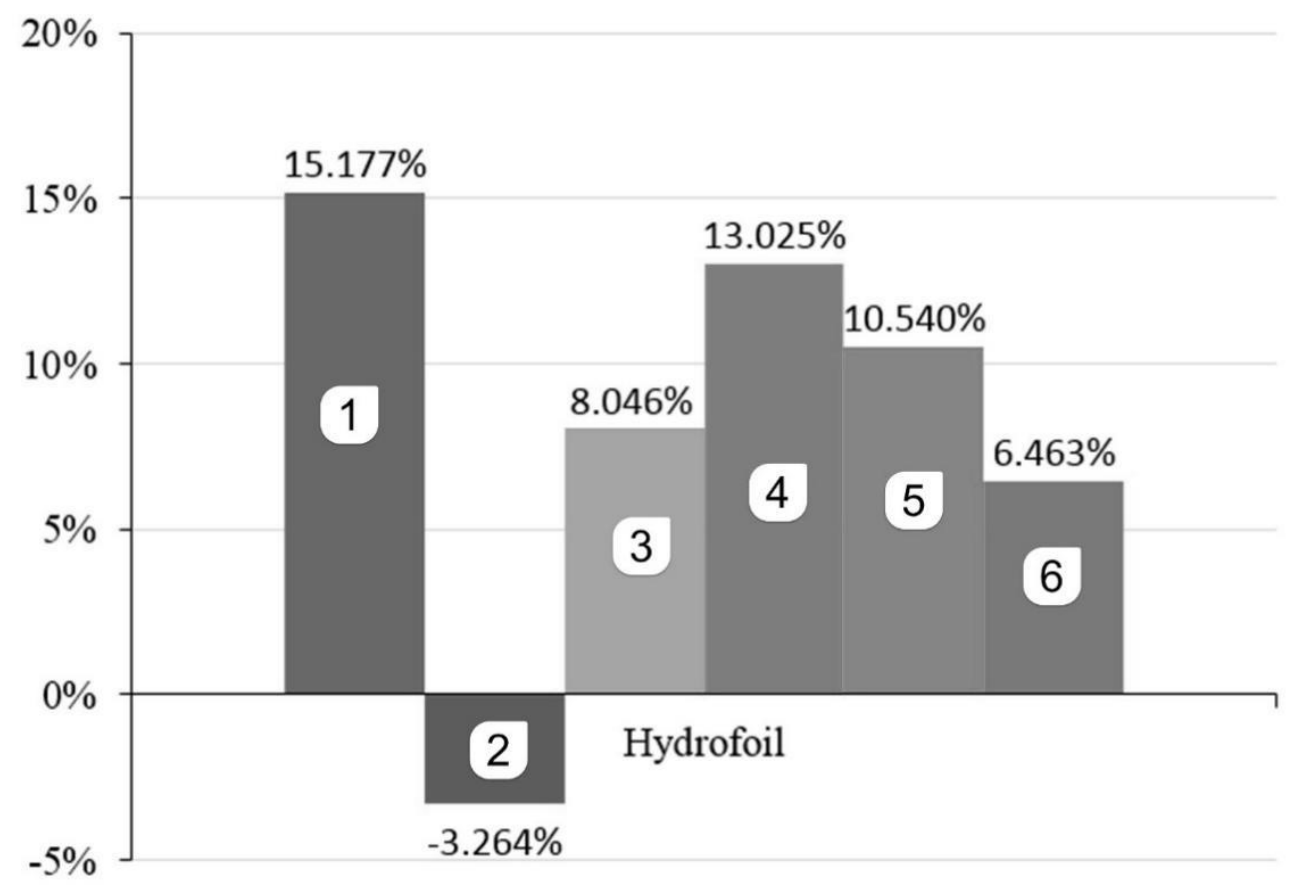

Fig.18. The thrust efficiency of the hydrofoils; the number in the column represents the hydrofoil's number

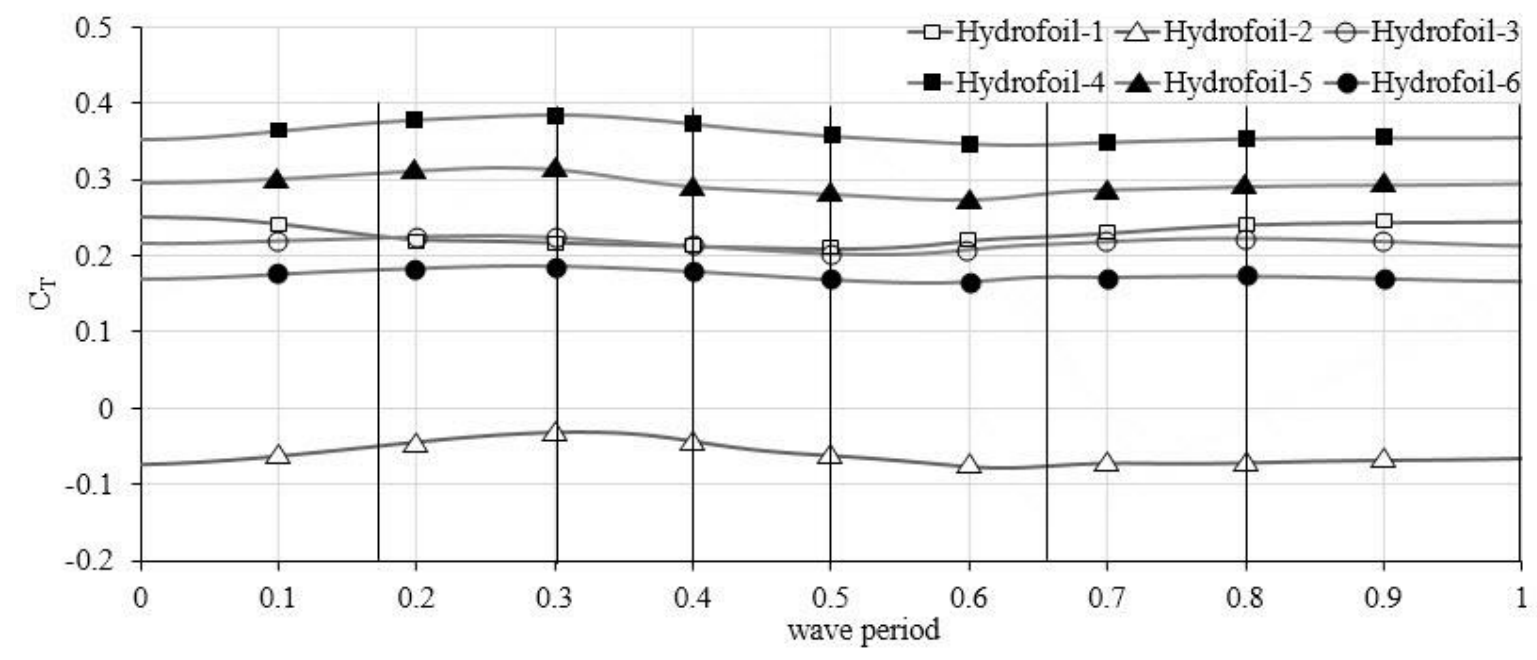

Fig.19. The average thrust coefficients of the hydrofoils. Location in the cycle is indicated by black vertical lines; it is represented eight times $(T=0,0.175,0.3,0.4,0.5,0.66,0.8,1.0)$ 


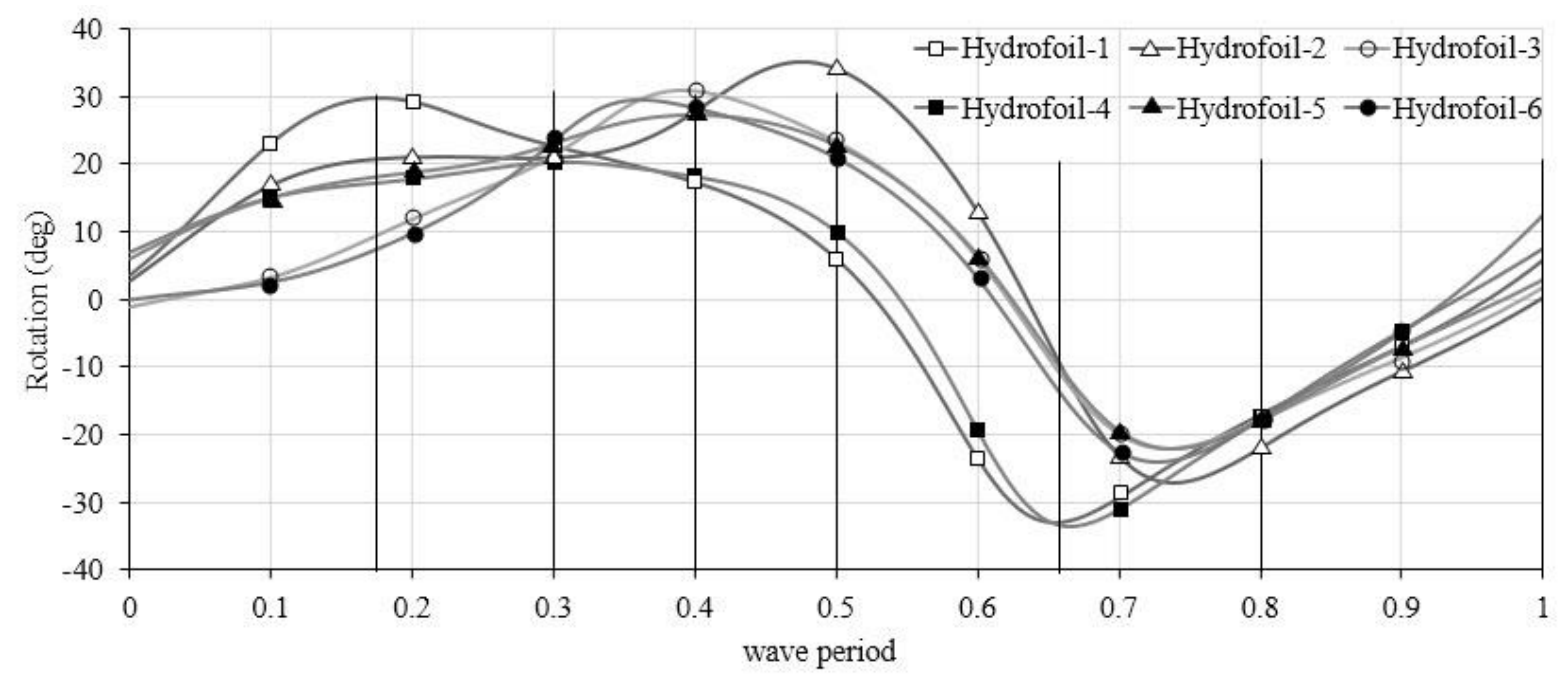

Fig.20. The rotation of the hydrofoils. Location in the cycle is indicated by black vertical lines; it is represented eight times $(T=0,0.175,0.3,0.4,0.5,0.66,0.8,1.0)$ 


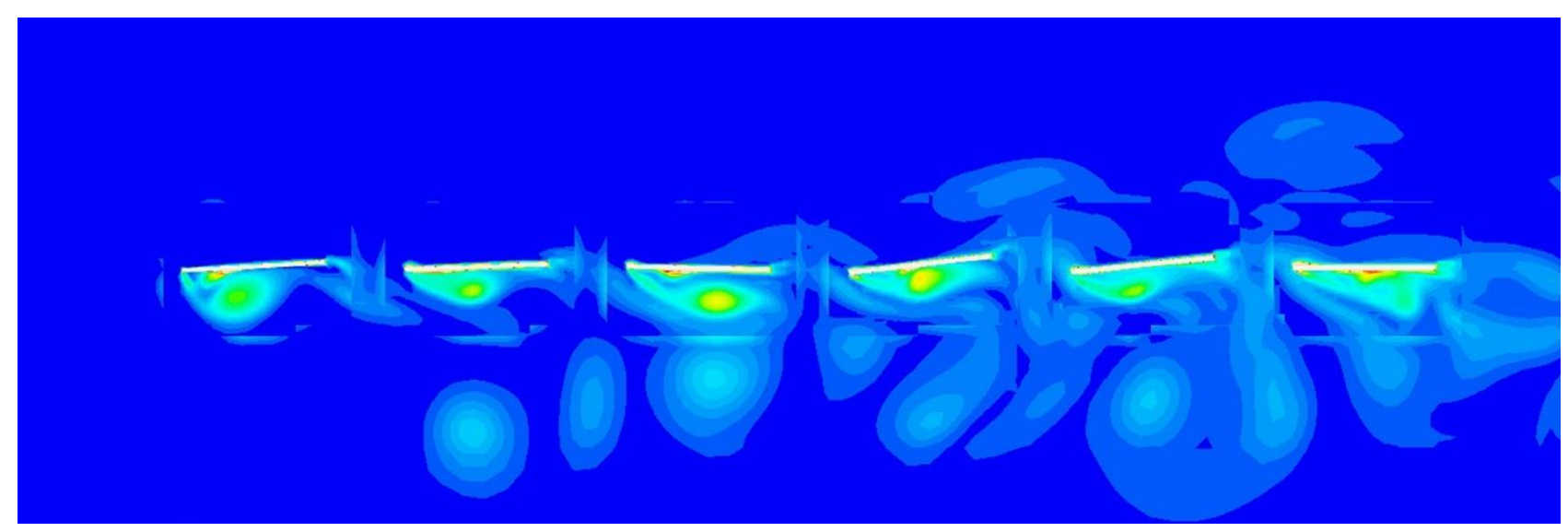

(a)

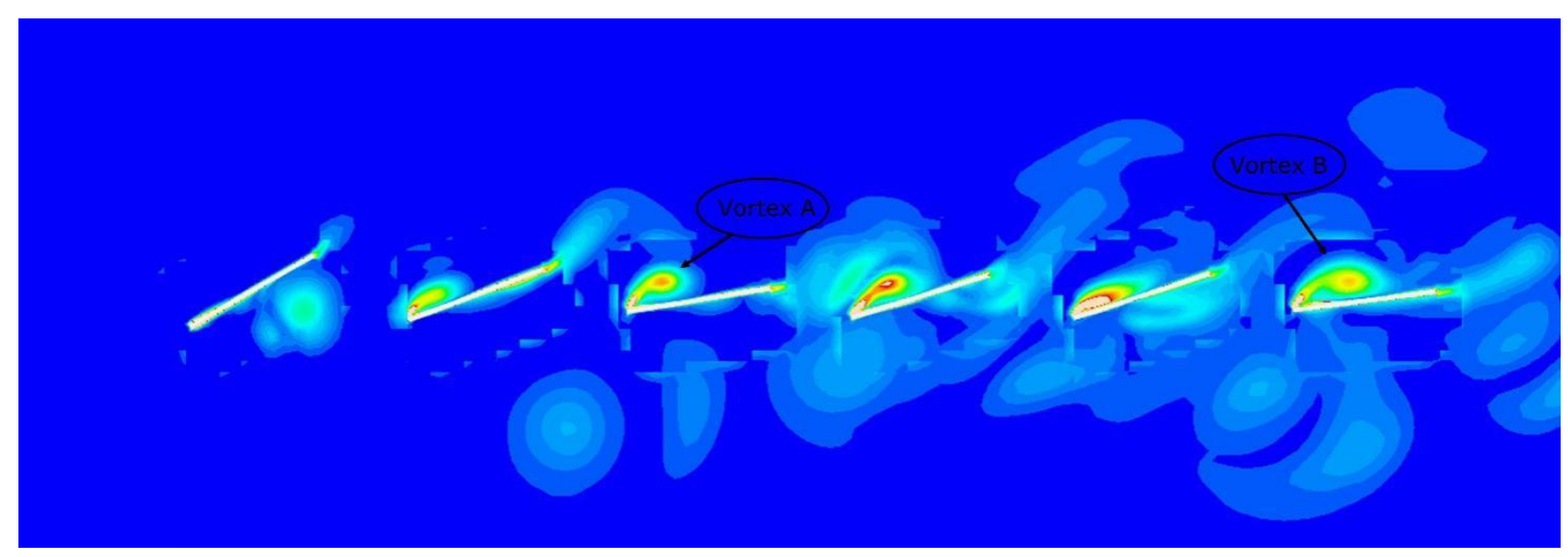

(b)

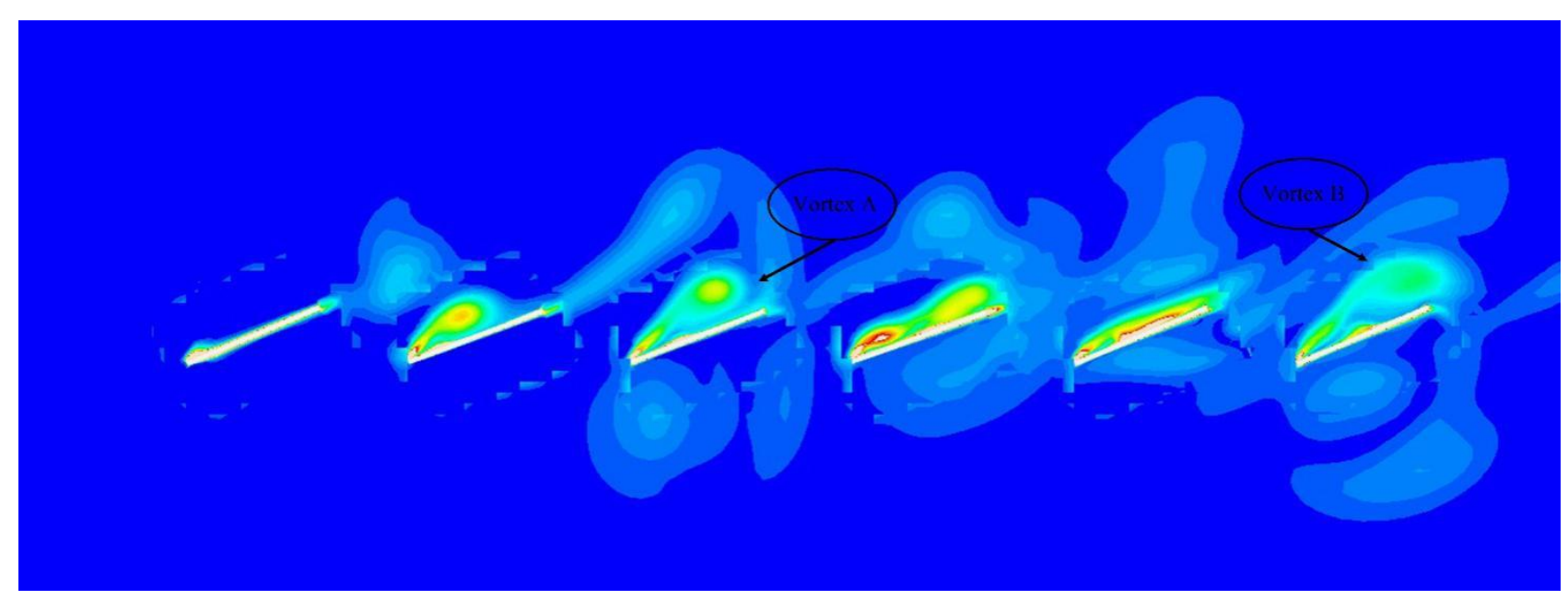

(c) 


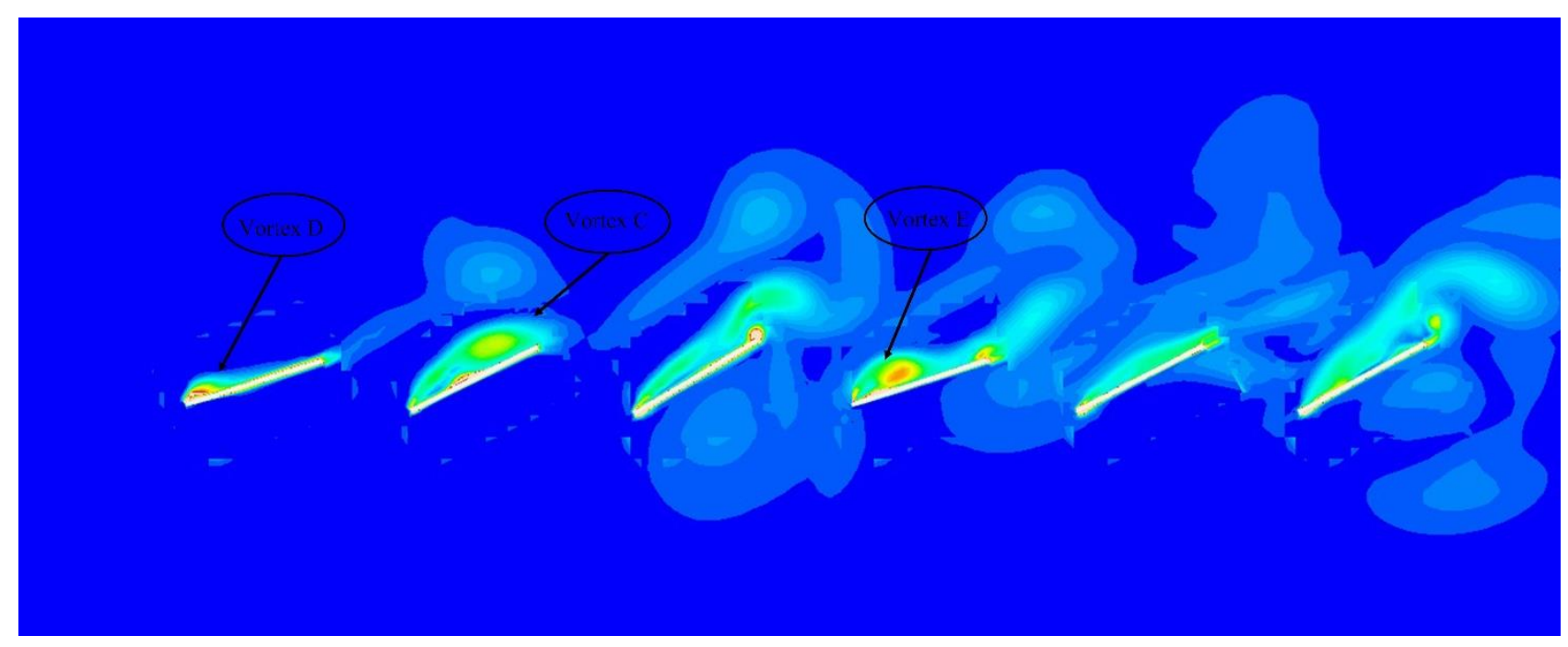

(d)

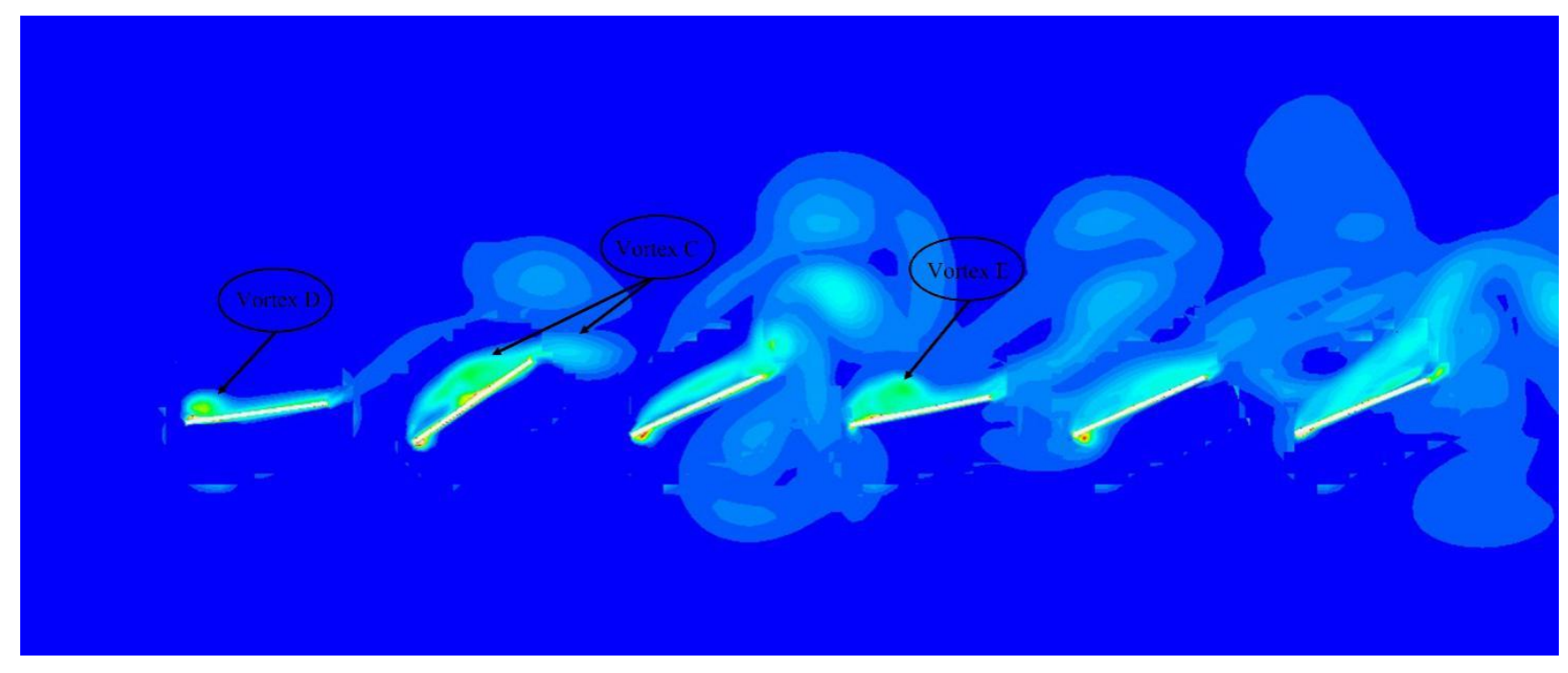

(e)

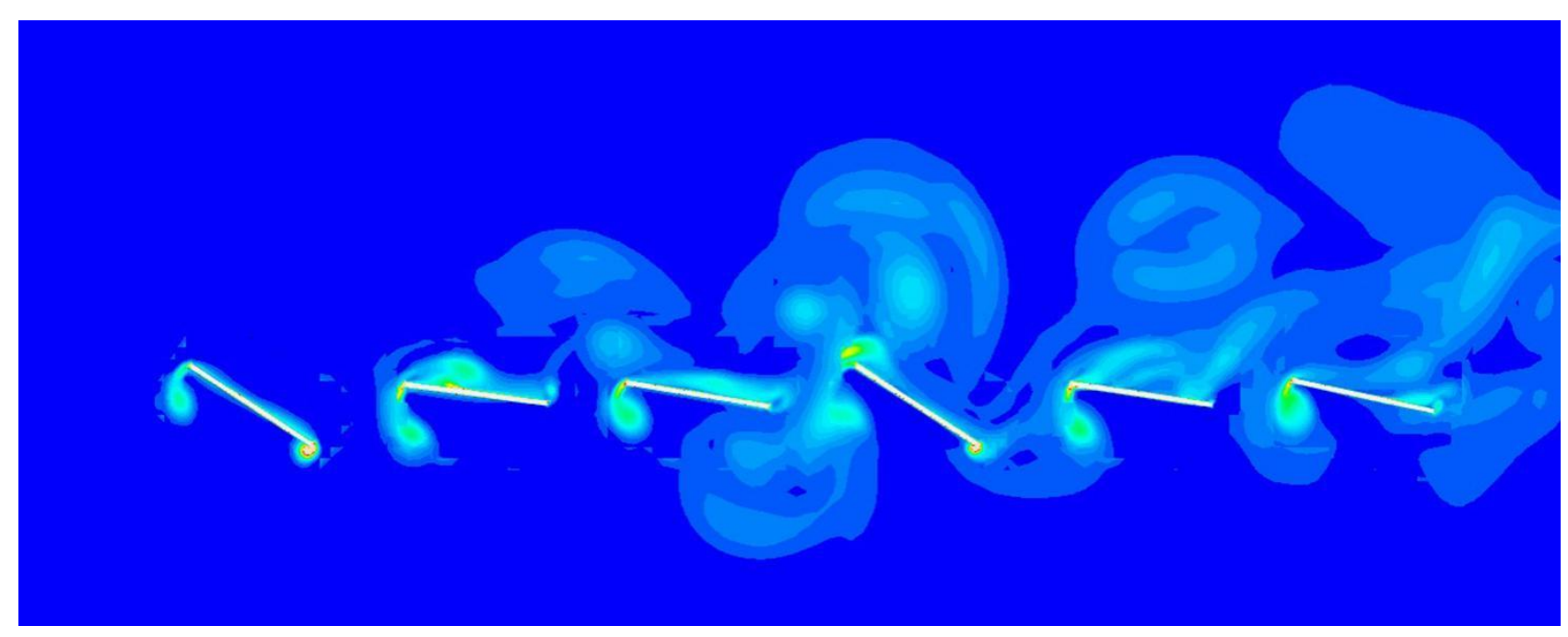




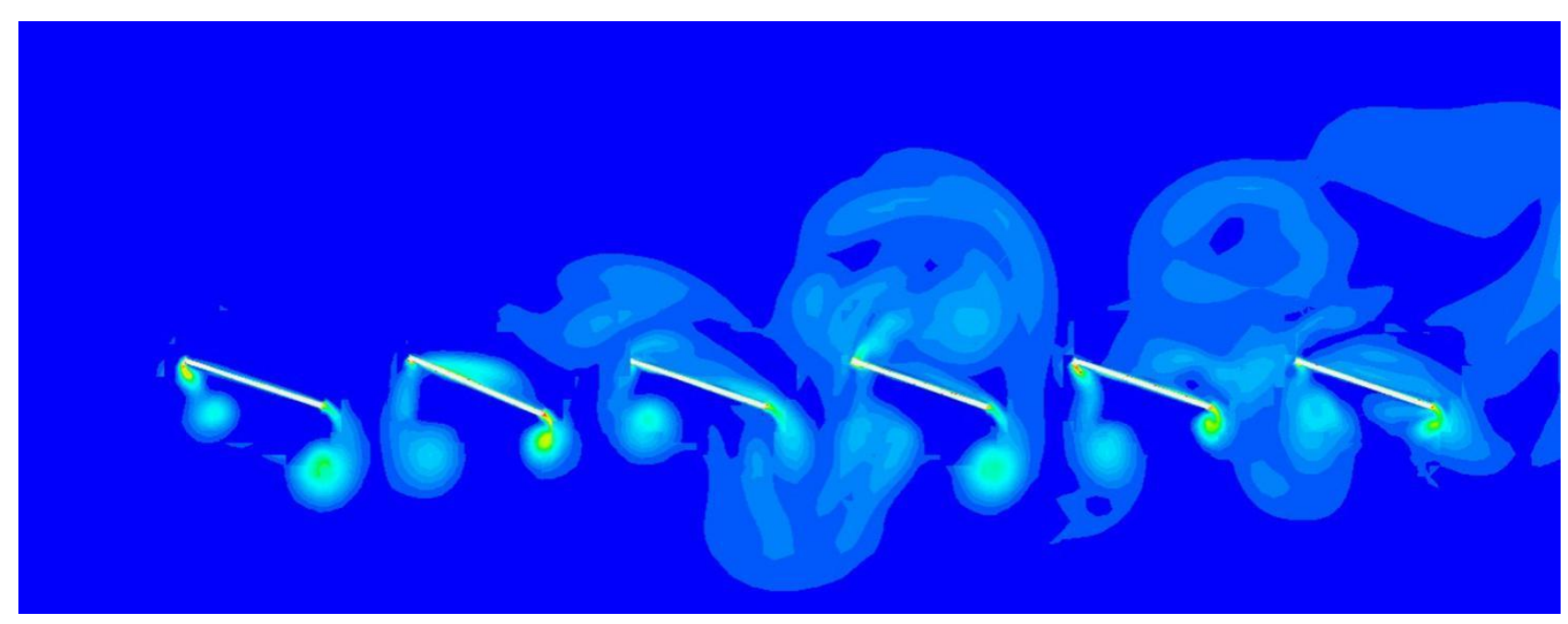

(g)

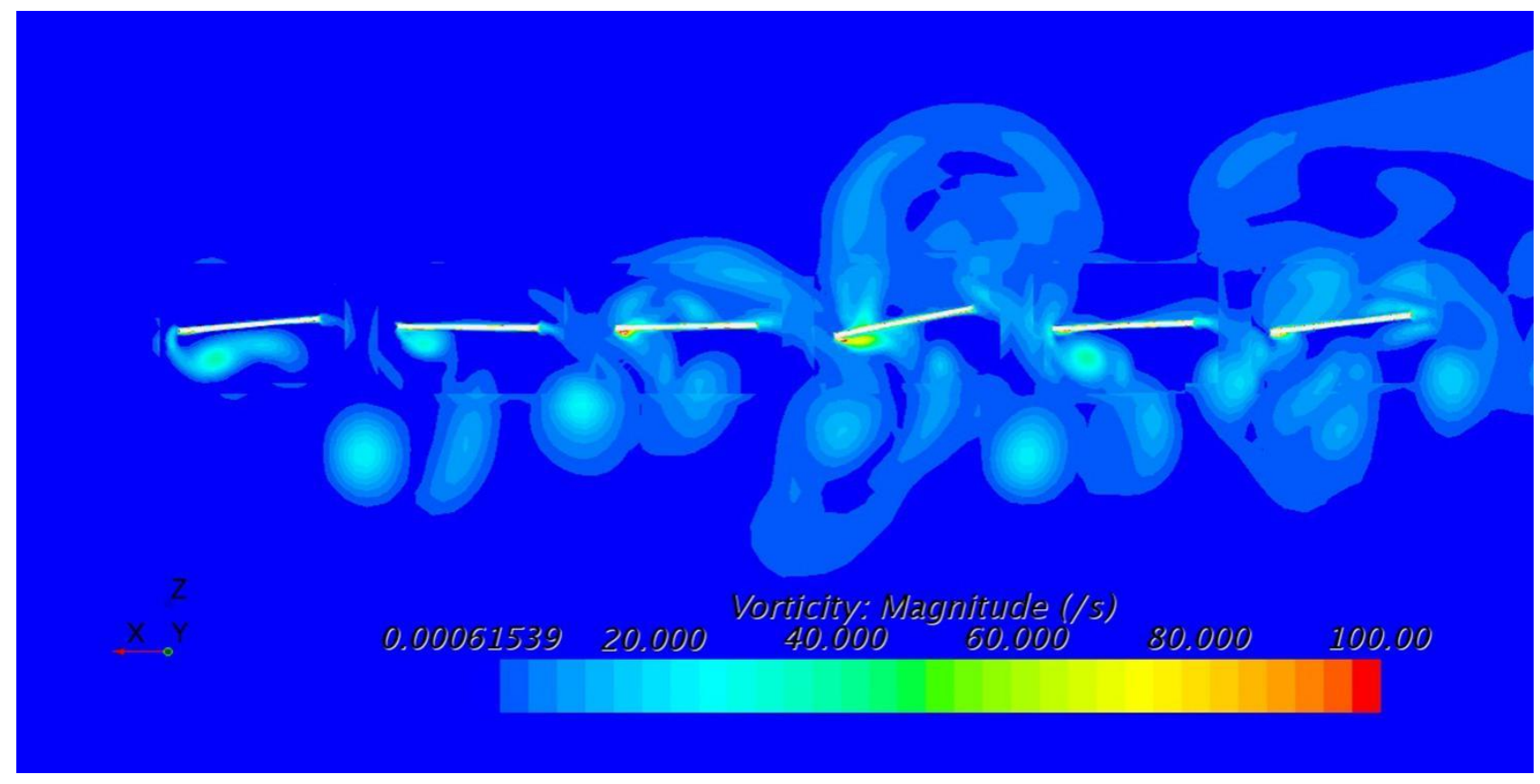

(h)

Fig.21. Time series of the vorticity magnitude development in one cycle of the hydrofoil (a) $\mathrm{T}=0$; (b) $\mathrm{T}=0.175$; (c) $\mathrm{T}=0.3$; (d) $\mathrm{T}=0.4 ;$ (e) $\mathrm{T}=0.5$; (f) $\mathrm{T}=0.66$; (g) $\mathrm{T}=0.8$; (h) $\mathrm{T}=1.0$; 
Table 1. The wave glider general parameters

\begin{tabular}{cc}
\hline Parameter & Full scale \\
\hline The length of the surface boat, $\mathrm{L}_{\mathrm{B}} / \mathrm{m}$ & 2 \\
The waterline length of the surface boat, $\mathrm{L}_{\mathrm{BWL}} / \mathrm{m}$ & 1.859 \\
The beam of the surface boat, $\mathrm{B} / \mathrm{m}$ & 0.6 \\
The draft of the surface boat, $\mathrm{d}_{\mathrm{B}} / \mathrm{m}$ & 0.115 \\
The freeboard of the surface boat, $\mathrm{df} \mathrm{f}_{\mathrm{B}} / \mathrm{m}$ & 0.105 \\
The displacement of the surface boat, $\Delta_{\mathrm{B}} / \mathrm{kg}$ & 50 \\
The length of the glider, $\mathrm{L}_{\mathrm{G}} / \mathrm{m}$ & 1.866 \\
The width of the glider, $\mathrm{B}_{\mathrm{G}} / \mathrm{m}$ & 0.02 \\
The height of the glider, $\mathrm{H}_{\mathrm{G}} / \mathrm{m}$ & 0.46 \\
The displacement of glider, $\Delta_{\mathrm{G}} / \mathrm{kg}$ & 40 \\
The length of the cable, $\mathrm{L}_{\mathrm{C}} / \mathrm{m}$ & 3 \\
The chord of the hydrofoil, $\mathrm{C} / \mathrm{m}$ & 0.16 \\
The span of the hydrofoil, $S_{h y} / \mathrm{m}$ & 0.6 \\
The thickness of the hydrofoil, $t_{h y} / \mathrm{m}$ & 0.004 \\
\hline
\end{tabular}

Table 2(a). Grid independence study for NACA0012 airfoil at Re $=5 \times 10^{5}$ and angles of attack of $10^{\circ}$ and $16^{\circ}$ in FINE/Marine

\begin{tabular}{|c|c|c|c|c|}
\hline \multirow[t]{3}{*}{ Number of cells } & \multicolumn{2}{|c|}{ Angle of attack 10} & \multicolumn{2}{|c|}{ Angle of attack 16} \\
\hline & Lift & Drag & Lift & Drag \\
\hline & Coefficient & Coefficient & Coefficient & Coefficient \\
\hline Grid 1: $\quad 68,812$ & 0.88184 & 0.05316 & 1.09374 & 0.08653 \\
\hline Grid 2: $\quad 178,686$ & 0.88963 & 0.04501 & 1.11291 & 0.08273 \\
\hline Grid 3: $\quad 296,750$ & 0.88655 & 0.04211 & 1.09572 & 0.09686 \\
\hline
\end{tabular}

Table 2(b). Grid independence study for NACA0012 airfoil at $\operatorname{Re}=5 \times 10^{5}$ and angles of attack of $10^{\circ}$ and $16^{\circ}$ in STAR-CCM+

\begin{tabular}{|c|c|c|c|c|}
\hline \multirow[t]{3}{*}{ Number of cells } & \multicolumn{2}{|c|}{ Angle of attack 10} & \multicolumn{2}{|c|}{ Angle of attack 16} \\
\hline & Lift & Drag & Lift & Drag \\
\hline & Coefficient & Coefficient & Coefficient & Coefficient \\
\hline Grid 1: $\quad 53,972$ & 0.89820 & 0.03884 & 1.12643 & 0.08252 \\
\hline Grid 2: $\quad 106,938$ & 0.89683 & 0.03893 & 1.12665 & 0.08251 \\
\hline Grid 3: $\quad 145,308$ & 0.89493 & 0.03815 & 1.13059 & 0.07947 \\
\hline
\end{tabular}


Table 3. The average thrust coefficient of the tandem hydrofoils in two-dimension and three-dimension

\begin{tabular}{ccccc}
\hline & \multicolumn{4}{c}{ Average thrust Coefficient } \\
\cline { 2 - 5 } & $\begin{array}{c}\text { Upstream foil } \\
\text { (Present study) }\end{array}$ & $\begin{array}{c}\text { Downstream foil } \\
\text { (Present study) }\end{array}$ & $\begin{array}{c}\text { Upstream foil } \\
\text { (Kinsey and Dumas } \\
(2012))\end{array}$ & $\begin{array}{c}\text { Downstream foil } \\
\text { (Kinsey and Dumas } \\
(2012))\end{array}$ \\
\hline Two-dimensional & 1.707 & 1.174 & 1.671 & 1.169 \\
$\begin{array}{c}\text { Three-dimensional } \\
(\text { AR=7) }\end{array}$ & 1.588 & 1.156 & 1.542 & 1.179 \\
\hline
\end{tabular}

Table 4. The average thrust force and average mechanical conversion power of the hydrofoils

\begin{tabular}{lcc}
\hline Hydrofoil & Average thrust force $(\mathrm{N})$ & Average mechanical conversion power(W) \\
\hline Hydrofoil-1 & 0.8038 & 1.3726 \\
Hydrofoil-2 & -0.2188 & 1.7372 \\
Hydrofoil-3 & 0.7043 & 2.2687 \\
Hydrofoil-4 & 1.1740 & 2.3359 \\
Hydrofoil-5 & 0.9711 & 2.3878 \\
Hydrofoil-6 & 0.5485 & 2.1995 \\
\hline
\end{tabular}

\section{校对报告}

当前使用的样式是 [Ocean Engineering by Ma]

当前文档包含的题录共29条

有 0 条题录存在必填字段内容缺失的问题

所有题录的数据正常 